\title{
LA e-INCLUSIÓN FEMENINA Y EL DESARROLLO SOCIOECONÓMICO EN EL ECUADOR: UNA PERSPECTIVA DE GÉNERO
}

\section{FEMALE e-INCLUSION AND SOCIOECONOMIC DEVELOPMENT IN ECUADOR: A GENDER PERSPECTIVE}

\begin{abstract}
Annie Muñoz Aroca, Msc. Máster en Desarrollo Económico y Políticas Públicas (España). Docente titular de la Universidad de Guayaquil, Ecuador. annie.munoza@ug.edu.ec

Olinda Sánchez Gordillo, Mgs. Magíster en Administración Bancaria y Finanzas (Ecuador) Docente investigadora de la Universidad de Guayaquil, Ecuador. olinda.sanchezg@ug.edu.ec

Francisco Arias Larrea, Mgs. Magíster en Dirección y Administración de Empresas (Ecuador). Docente de planificación de la Universidad de Guayaquil, Ecuador. francisco.ariasl@ug.edu.ec

\section{Lupe García Espinoza, Ph.D.} Doctora en Ingeniería agroforestal (España). Docente Ocasional de la Universidad de Guayaquil, Ecuador. lupe.garcia@ug.edu.ec
\end{abstract}

\section{ARTÍCULO DE INVESTIGAIÓN}

Recibido: 5 de junio de 2018.

Aceptado: 27 de junio de 2018. 


\section{RESUMEN}

Este artículo aborda la incidencia de las tecnologías de la información y la comunicación (TIC) en el desarrollo social del Ecuador, desde una perspectiva de género. Su objetivo principal, fue determinar si el uso y acceso a las computadoras e internet influye en gran medida a la hora de favorecer el éxito académico, la generación de empleo, obtención de salarios más altos y elevar niveles de competitividad. Para llevar a cabo esta investigación se recurrió a información estadística disponible sobre las diferencias de género en relación a las TIC, además se realizó una encuesta en línea, evidenciándose que los estereotipos sociales poseen gran influencia sobre el desenvolvimiento de la mujer en la sociedad. Asimismo, se determinó que a pesar de que existe disparidad en cuanto al acceso a las tecnologías de la información por parte de las mujeres, el uso que se puede dar a las mismas desde la perspectiva femenina posee un alto grado de funcionalidad y contribución al desarrollo social. Así se plantea la promoción de la e-inclusión como una política pública de desarrollo económico y social en el Ecuador.

Palabras clave: Género, desarrollo social, tecnologías de la información, inclusión digital.

\section{ABSTRACT}

This article deals with the incidence of information and communication technologies (ICT) in the social development of Ecuador, from a gender perspective; Its main objective was to determine whether the use and access to computers and the internet has a great influence on promoting academic success, generating employment, obtaining higher salaries and raising levels of competitiveness. To carry out this research, available statistical information was used on gender differences in relation to ICT, showing that social stereotypes have great influence on the development of women in society; It was also determined that although there is a disparity in access to information technologies by women, the use that can be given to them from the female perspective has a high degree of functionality and contribution to development. Social. This is how the promotion of e-inclusion is suggested as a public policy of economic and social development in Ecuador.

Keywords: Gender, social development, information technologies, digital inclusion. 


\section{INTRODUCCIÓN}

El desarrollo científico tecnológico en el contexto de la globalización ha dado muestras de importantes brechas que afectan de forma diferenciada a hombres y mujeres según los contextos a los que se refieren; en cuanto a las tres brechas reconocidas, esto es: acceso a material, usos y habilidades y usos avanzados, es en estas dos últimas en donde las mujeres se encuentran en franca desventaja y esto se debe básicamente a restricciones sociales entre las que destacan las asociadas al género (Castaño, 2013). En Ecuador, por ejemplo, para el año 2016 son más los hombres que utilizan el internet para temas laborales (38,6\% y $37 \%$ respectivamente) (INEC, 2017).

Si bien a nivel de país los indicadores relacionados con las TIC, han mejorado considerablemente, no se puede desconocer que se mantienen sustanciales brechas de carácter territorial, generacional y por sexo, las cuales tienen incidencia en el desarrollo nacional. Las causas de que las mujeres son excluidas del acceso tecnológico están relacionadas con su condición económica, ya que en condiciones de pobreza la participación de nuevas tecnologías es mucho más difícil, sin embargo, son factores asociados al género y no otros los que en muchos casos explican su inaccesibilidad.

En Ecuador, las causas por las cuales las mujeres no participan en condiciones de igualdad de las nuevas tecnologías son principalmente en lo que se refiere a la determinación del uso que se le da, el tiempo asignado para su uso y el aprovechamiento de esta información para configurar estrategias que permitan alcanzar condiciones, así como reducir las brechas sociales, económicas, culturales y laborales; están relacionadas con el ingreso, el grado de educación y los estereotipos de género que todavía direccionan a las mujeres hacia oficios y profesiones consideradas femeninas (CEPAL, 2016 citado en Botello, 2015).

Esta condición no solamente explica el orden mundial actual, o marca la diferencia entre países, regiones o continentes, sino que también es una variable muy tomada en cuenta a la hora de medir los niveles de desarrollo y subdesarrollo al interior de los países; el acceso a las nuevas tecnologías marca también la diferencia entre localidades, instituciones y personas, las estadísticas mundiales son claramente la evidencia de aquello. 
La Planificación Nacional para el desarrollo en Ecuador, contempla en algunos de sus objetivos, estrategias, políticas y lineamientos el cierre de brechas de desigualdad de género, así como la erradicación de todas las formas de discriminación y exclusión aún presentes en el país (SENPLADES, 2014). De esta manera, hasta la presente fecha, se han logrado avances significativos al respecto; sin embargo, la fuerza que ejercen las tradiciones culturales y los estereotipos sociales en general, juegan un papel muy importante a la hora de encaminar esas políticas hacia el desarrollo social del país.

Es necesario acotar que en Ecuador, el área de las mujeres y TIC es un tema escasamente abordado en los estudios socioeconómicos, principalmente en lo que se refiere a la determinación del uso que se le da por parte del género femenino a las nuevas tecnologías, el tiempo asignado para su uso y el aprovechamiento de esta información para configurar estrategias que permitan reducir las brechas sociales, económicas, culturales y laborales con un enfoque de productividad y competitividad económica para el país.

En el resto de países de América Latina, la situación no es muy diferente; no obstante, hay un estudio realizado por la Asociación Global de todo el Ecosistema Móvil - GSMA (2016), que indica que "la encuesta al consumidor no revela una brecha importante de género en la región respecto a la adopción de internet.", este resultado, es claramente la evidencia que las desigualdades no se encuentran en el acceso, sino más bien en la incorporación femenina en los roles de toma de decisión, el incentivo a su participación activa en el diseño de políticas públicas o a seleccionar carreras de estudio que no se encuentren en el catálogo de carreras que "deberían" estudiar las mujeres, así como la potenciación de sus capacidades en beneficio del desarrollo científico y económico. Es irónico evidenciar, a través de las estadísticas, que alrededor del mundo existen miles y millones de mujeres altamente capacitadas técnica y científicamente para enfrentar situaciones con alto grado de complejidad que afectan a las sociedades, pero es el mundo el que no esté preparado para asignar el rol adecuado a cada una de ellas.

El objetivo principal de esta investigación, está en determinar si el uso y acceso a las computadoras e internet influye en gran medida a la hora de favorecer el éxito académico, 
la generación de empleo, obtención de salarios más altos y elevar niveles de competitividad; además procura evidenciar si existen otros factores relacionados, que influyan en la pervivencia de brechas de desigualdad de género y que podrían estar abonando a la distribución desigual de oportunidades profesionales.

\section{REVISIÓN TEÓRICA}

Según Aponte (2008) en América Latina y el Caribe, el aumento de la matrícula femenina en educación de pregrado no ocurre del mismo modo que en todas las áreas del conocimiento, se puede notar menor participación femenina en carreras relacionadas con las ciencias naturales (física, química y matemáticas), ingeniería y tecnología, agronomía, medio ambiente y construcción, arquitectura y artes gráficas y se percibe una preferencia a cursar carreras de las áreas de ciencias sociales, humanidades y ciencias de la salud. La razón por la cual la matrícula femenina sigue esta tendencia se encuentra más en valoraciones culturales que de otra índole; en todo caso las evidencias confirman que, por intereses, confianza, auto concepto y entrenamiento diferencial, las mujeres no se inclinan por estudios científicos-tecnológicos (González, et al. 2016).

Para Aznar y Navarro (2012) "la elección de los estudios, ya sean en ciclos formativos o en la universidad tiene consecuencias en las posteriores diferencias en su inserción y posterior evolución en el mercado laboral derivando en una auténtica segregación horizontal del mercado de trabajo" (p.16). Así en Brasil, por ejemplo, se encuentra que a pesar de que las mujeres mejoraron su ubicación en empleos asociados con las TIC, el ingreso al mismo se dio a través de la tercerización económica y la economía de servicios, persistiendo la segregación salarial (Berrió, et al. 2017, p. 181).

Bethencourt (2012) señala que el género también estructura una división dentro del trabajo remunerado entre funciones y posiciones más valorizadas que ocupan a una mayor proporción de hombres, y aquellas de menor prestigio y reconocimiento, donde se concentran mayoritariamente las mujeres. Por tanto, para complementar la idea anterior, García (2015) menciona que los estereotipos, además de estructurar la realidad, sirven para justificar el prejuicio y la discriminación hacia ciertos grupos sociales como un símbolo de 
superioridad o emancipación. En este mismo sentido, Torres (2012) señala que no es aventurado afirmar que las mujeres no están consolidadas como sujetos productivos/económicos o por lo menos, no en el mismo sentido que los hombres, considerando que este es el modelo a seguir en la cotidianidad de la vida. Indica además que las mujeres en el ámbito laboral todavía son conceptualizadas como menos disponibles que los hombres ya que desde la óptica económica/patriarcal y estereotipada, ellas no son sujetos económicos/productivos, en vista de que este sujeto se cataloga como plenamente disponible, es decir, un sujeto sin compromisos personales, ausente de vinculaciones familiares (al menos en abstracto), por tanto, un sujeto varón. Las mujeres, desde este imaginario, siempre son vistas como madres (o futuras madres), como sujetos sustituibles o reemplazables. Como sujetos, en suma, cuya vinculación profesional y/o laboral siempre va a estar condicionada por la capacidad reproductiva de su sexo biológico y por su dedicación al cuidado de los demás en el ámbito familiar.

La fase expansiva del capitalismo y de la revolución industrial son algunos de los momentos históricos que han contribuido significativamente a la subordinación de la mujer en la sociedad. A lo largo de la historia los movimientos feministas han luchado con éxito por la inclusión de la mujer en lo público, sus derechos económicos, acceso a la educación, entre otros, aunque su inserción en la vida pública ha sido lenta y limitada, ya que al situar la fuente de trabajo lejos del hogar familiar, se acentuó la separación entre las esferas del hogar y del trabajo, por lo que se redujeron las posibilidades de la mujer de participar en la vida laboral. Lo anterior remitió a las mujeres a la vida doméstica y esta segregación, de algún modo, se tradujo en la subordinación de las mujeres a limitarse al ámbito privado y no participar en lo público, ni en lo económico (Arredondo, Maldonado y Velázquez, 2013).

Desde el enfoque de desarrollo, se han creado (sin éxito) una multitud de instituciones encargadas del desarrollo de la mujer, con el fin de que abandonen su condición de subalternas en el tejido institucional nacional e internacional. Hoy en día existen muchos indicadores que buscan medir la situación de la mujer, sin embargo, muy poco se aborda este aspecto en relación con las relaciones patriarcales de poder al interior de la familia, que condicionan todo acceso de las mujeres a otros ámbitos económico o político. Por otro lado, las ciencias económicas siguen ignorando la dimensión de género y la productividad 
del trabajo de cuidado, manteniendo al PIB como indicador maestro de su orientación (Aguinaga et al. 2012).

Una de las situaciones que pueden impulsar el desarrollo de la mujer, es la incorporación de su creatividad para el emprendimiento. Para Buitrago y Duque (2013), el escritor y especialista John Howkins define la economía creativa como la comprensión de los sectores en los que el valor de sus bienes y servicios se fundamenta en la propiedad intelectual: arquitectura, artes visuales y escénicas, artesanías, cine, diseño, editorial, investigación y desarrollo, juegos y juguetes, moda, música, publicidad, software, TV y radio, y videojuegos.

La Economía creativa o Economía Naranja representa una riqueza enorme basada en el talento, la propiedad intelectual, la conectividad y por supuesto, la herencia cultural de la región. Este modelo económico podría convertirse en una solución para aplicarlo en las mujeres con la finalidad de que sean las gestoras de su propio desarrollo, con su aplicación en el conocimiento, cultura y talento posiblemente no explorados y explotados.

Para Castaño y Webster (2014) el género se proyecta en una concepción excesivamente masculina y tecnológica de la innovación, centrada en el progreso de la alta tecnología, mientras toda innovación "femenina" y "social" queda relegada a un segundo puesto, además menciona que las mujeres se concentran en áreas de trabajo de baja remuneración y su presencia es todavía escasa en las ocupaciones profesionales y mejor pagadas, con el resultado de que su potencial está infrautilizado y su contribución a la ciencia y la tecnología no llega a realizarse de forma completa (p.24).

Versozi, Muñoz y Versozi (2017) señalan que hacen falta más esfuerzos desde todos los sectores, a pesar de que en los últimos años los ecuatorianos han alimentado su interés por adquirir nuevas tecnologías y tener acceso a la información, se desconoce totalmente el uso adecuado que se les esté dando a estos recursos tecnológicos y mucho menos se conoce el tipo de información que se acuna en los hogares a través del internet. 
El término recientemente acuñado de nueva economía o economía inmaterial, es decir, el desarrollo de toda la economía basada en la información y el conocimiento, y no en la producción de bienes industriales, está llamada a ser la gran especialización de los países desarrollados frente al resto del mundo en el siglo XXI. La economía del conocimiento, entendida como la utilización de información de valor en las actividades de los distintos sectores de la economía, puede llegar a representar un nuevo modelo económico sostenible a medio y largo plazo que ayude a superar la crisis actual (Versozi, et. al 2017, p. 482).

Desde la perspectiva económica, en cuanto al aporte de las TIC al PIB del Ecuador, se observa que su contribución está en el orden del 2\% del ingreso nacional para el año 2013, según información del Banco Central del Ecuador (Ministerio de Telecomunicaciones y Sociedad de la Información, 2016, p. 17).

En cuanto a nivel regional se refiere, los países de América Latina y el Caribe (ALC) han cohesionado esfuerzos en la eLAC, que según la CEPAL en su página web la define como:

Una plataforma de diálogo político que reúne a las autoridades de los ministerios e instituciones encargadas de las agendas digitales y de las políticas sobre las tecnologías de la información y las comunicaciones (TIC), así como a representantes del sector privado, la academia y la sociedad civil (CEPAL, 2015).

Una forma sencilla de medir el estado actual y el desarrollo de la banda ancha en ALC, es a través del Índice de Desarrollo de la Banda Ancha (IDBA). Al respecto, el Banco Interamericano de Desarrollo (BID) indica que:

El IDBA evalúa un elemento muy concreto y a la vez esencial para la sociedad de la información: el desarrollo de la banda ancha. Además, a partir de las variables que lo componen, se ofrecen otras cuatro medidas de desarrollo específicas que son los pilares sobre los que se asienta el desarrollo de la banda ancha:

- Políticas Públicas y Visión Estratégica 
- Regulación Estratégica

- Infraestructuras

- Aplicaciones y Capacitación (BID, 2017, p. 14).

\section{MATERIALES Y MÉTODOS}

Para realizar el estudio sobre el alto grado de importancia que tiene la mujer en el desarrollo socioeconómico del Ecuador, a través de la potenciación de sus capacidades y la optimización de las nuevas tecnologías se utiliza una revisión teórica y sistemática, tomando en consideración esta última se utilizaron cuatro etapas de la práctica basada en evidencias aplicadas generalmente en investigaciones médicas:

Tabla 1: IBE

$$
\begin{aligned}
& \text { Identificación de un problema } \\
& \hline \text { Formulación de un asunto relevante y específico } \\
& \hline \text { Búsqueda de datos o evidencias científicas } \\
& \hline \text { Evaluación de los datos disponibles }
\end{aligned}
$$

Fuente: Elaboración propia a partir de Mámedio da Costa, De Mattos, \& Cuce (2007)

Estas etapas representan la parte del estudio definida como revisión sistemática, en la que se sintetizan datos y se realizan estudios primarios.

Para la construcción y búsqueda de la pregunta de investigación se utilizó la herramienta PICO:

Tabla 2: PBE

\begin{tabular}{lll}
\hline P & Población & Mujeres \\
\hline I & Intervención & Discriminación \\
\hline C & Comparación & Perspectiva de género: \\
& & Hombres versus mujeres. \\
\hline
\end{tabular}




\begin{tabular}{ll}
\hline O Outside - desenlace & Resultados de información \\
& estadística que evidencia la falta \\
& de políticas públicas que \\
& garanticen la e-inclusión con \\
& fines de desarrollo \\
& socioeconómico a través del \\
& aprovechamiento del potencial \\
& femenino.
\end{tabular}

Fuente: Elaboración propia.

Preguntas de investigación construidas a partir de la herramienta PICO:

1. ¿Cuáles son las carreras afines a tecnologías de información por la que optan las mujeres?

2. ¿Cuál es la ocupación y actividad a la que se dedican las mujeres de acuerdo a su formación y cuáles son sus habilidades?

3. ¿En qué se distribuye el uso del tiempo según el sexo?

4. ¿Los estereotipos sociales influyen en el rol que ocupa la mujer en la sociedad, en cuanto al aspecto laboral, político, educativo, entre otros?

Pregunta de discusión:

5. ¿Políticas de e-inclusión como medida paliativa?

Los datos estadísticos donde se analiza la preferencia de las ramas de estudio por género, se obtuvieron de los registros de matriculación de la Universidad Central del Ecuador (Quito) y de la Universidad de Guayaquil (Guayaquil), considerando que son dos universidades que acogen estudiantes provenientes de todas las provincias del Ecuador.

Para determinar info-habilidades y e-experiencia en el uso de internet, se determinó el tamaño de la muestra con una población total de 16’000.000 de habitantes que tiene el 
Ecuador, se aplicó la fórmula estadística para una distribución normal con una probabilidad de fracaso del $50 \%$, nivel de confianza del $95 \%$ y un margen de error del $5 \%$.

$$
n=\frac{N \times Z_{a}^{2} \times p \times q}{d^{2} \times(N-1)+Z_{a}^{2} \times p \times q}
$$

Aplicada la fórmula se obtuvo que la muestra debía considerar a 384 personas, la muestra tomada para el estudio cubrió a 398 personas, la población objetivo se centró en estudiantes universitarios y profesionales de todas las edades a nivel nacional. Para ello se utilizó publicidad pagada por redes sociales (ubicación geográfica: nivel nacional, población objetivos: estudiantes de tercer nivel en adelante y profesionales), además se envió la encuesta a familiares o amigos que cumplían con el perfil, los resultados fueron sometidos a una selección y se utilizaron datos equitativos pero aleatorios para todas las regiones del país (Costa, Sierra, Amazonia y Región Insular).

La ejecución de la encuesta se realizó a través de un aplicativo on-line y el link fue compartido y enviado a diferentes ciudades del Ecuador a través de las redes sociales más conocidas. El total de datos obtenidos fue clasificado por sexo y mostrado en porcentaje.

\section{ANÁLISIS DE LOS RESULTADOS}

Bajo los cuatro pilares enfocados por el índice de Desarrollo de Banda Ancha, se presenta en la figura 1, la comparación del Índice de Desarrollo de la Banda Ancha entre los países de la OCDE y ALC para el año 2016. Las variables de Infraestructuras y Aplicaciones y Capacitación muestran una variación porcentual del 1,91\% y 2,13\% respectivamente, estas variables son las que más diferencias tienen entre los países del ALC y OCDE. En cuanto a las variables de Políticas y Visión Estratégica y Regulación Estratégica, la variación porcentual está en el orden de $1,88 \%$ y $1,47 \%$ respectivamente. 


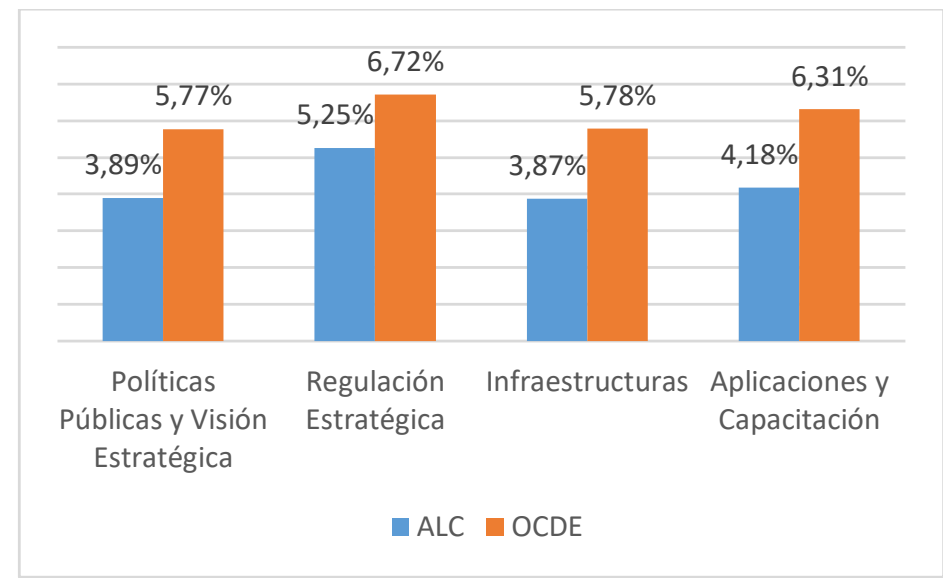

Figura 1. Índice de Desarrollo de la Banda Ancha 2016. Comparación entre los países del BID y OCDE.

Fuente: Elaboración propia a partir de datos obtenidos en el sitio web de DigiLAC $^{1}$ (BID, 2018).

Por otro lado, en cuanto a conectividad se refiere en América Latina, el número de hogares conectados a Internet, "creció en un 103\% entre el año 2010 y el 2016. Pese a ello, más de la mitad de los hogares siguen sin acceso a Internet” (CEPAL, 2018, pág. 7).

Si se analiza la brecha digital, entre países de la OCDE y ALC, se puede palpar una disminución de 10 puntos porcentuales, es decir una diferencia de 50,8\% en 2010 y $40,8 \%$ en 2016. Ver tabla 1.

Tabla 3. Variación porcentual de hogares con Internet, 2016.

\begin{tabular}{cccc}
\hline Años & OCDE & América Latina y el Caribe & $\Delta \%$ \\
\hline $\mathbf{2 0 1 0}$ & $73,2 \%$ & $22,4 \%$ & $50,8 \%$ \\
$\mathbf{2 0 1 1}$ & $75,4 \%$ & $28,0 \%$ & $47,4 \%$ \\
$\mathbf{2 0 1 2}$ & $77,7 \%$ & $31,8 \%$ & $45,9 \%$ \\
$\mathbf{2 0 1 3}$ & $81,3 \%$ & $35,5 \%$ & $45,8 \%$ \\
$\mathbf{2 0 1 4}$ & $82,5 \%$ & $39,7 \%$ & $42,8 \%$
\end{tabular}

\footnotetext{
1 DigiLAC es una plataforma virtual creada por el BID para cerrar las brechas existentes en la penetración de banda ancha y consolidar, a través de índices de desarrollo digital innovadores, mapas e informes de infraestructura, información clave sobre los desafíos, oportunidades y posibilidades de desarrollo de banda ancha en América Latina y el Caribe. Tomado de https://www.iadb.org/es/mercados-financieros/conectividad (BID, 2018).
} 


\begin{tabular}{llll}
$\mathbf{2 0 1 5}$ & $84,2 \%$ & $42,5 \%$ & $41,7 \%$ \\
\hline $\mathbf{2 0 1 6}$ & $86,3 \%$ & $45,5 \%$ & $40,8 \%$ \\
\hline
\end{tabular}

Fuente: Elaboración propia a partir del Estado de la banda ancha en América Latina y el Caribe 2017. (CEPAL, 2018)

Desde la perspectiva de género, a nivel de la región, el estudio del BID (2018) indica que: "la diferencia en cuanto a usuarios de Internet en promedio de solo 0,6 p.p. Sin embargo, la situación varía de país a país" (pág. 24), en el caso de Ecuador la diferencia de usuarios entre hombres y mujeres es relativamente superior a 50 p.p, siendo que la diferencia sea mínima a favor de los hombres (Figura 2).

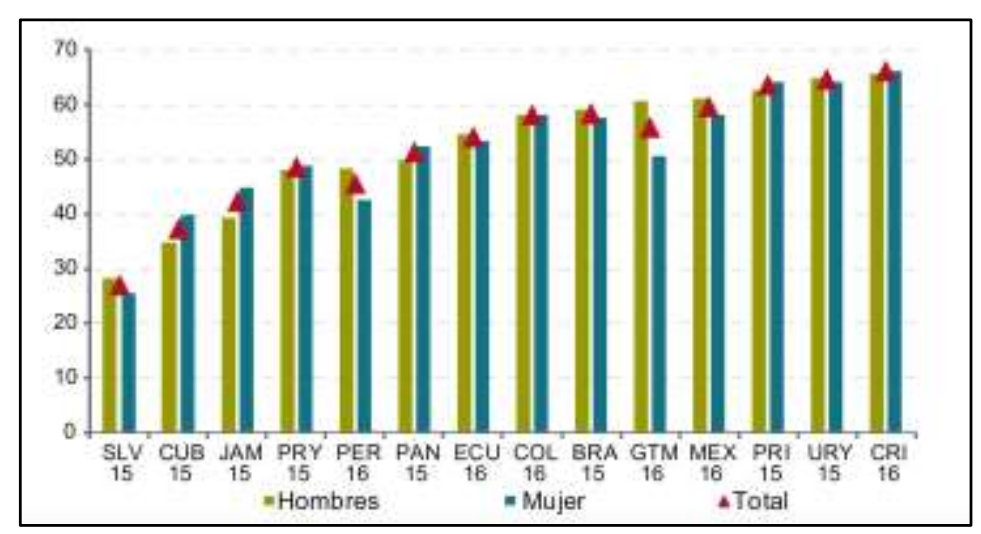

Figura 2. Usuarios de Internet según género, año más cercano al 2016, en porcentajes Fuente: Estado de la banda ancha en América Latina y el Caribe 2017 (CEPAL, 2018, p. 24)

Como se puede observar en la figura 3, el Estado Ecuatoriano ha tomado las pautas para tratar de acortar la brecha digital, con sentido de universalización de las tecnologías con carácter inclusivo y con pertinencia de género. 


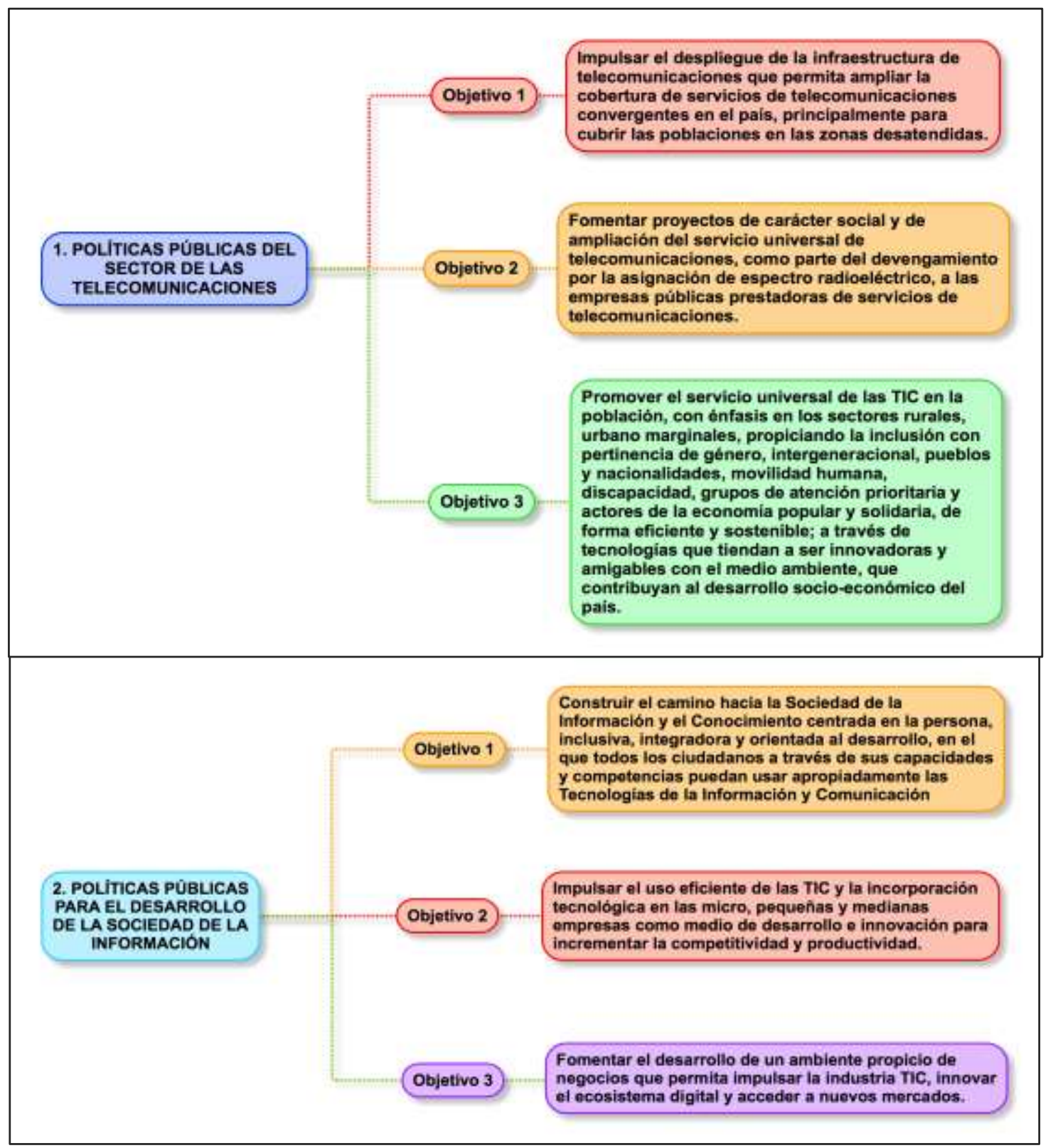

Figura 3. Políticas Publicas del Sector de las Telecomunicaciones y de la Sociedad de la Información 2017 - 2021.

Fuente: Elaboración propia a partir del Registro Oficial \# 15, 011-2017, 15 de junio del 2017. 


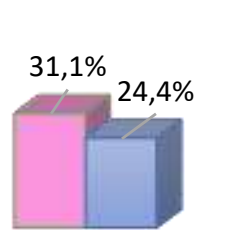

Nacional

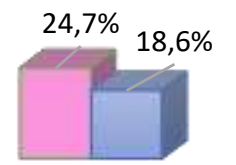

Urbana

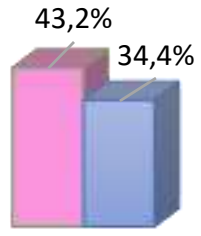

Rural

$\square$ Mujeres

Figura 4. Analfabetismo digital por área, población de 15 años y más. Tomado de Mujeres y hombres del Ecuador en cifras III.

Fuente: Obtenido del Instituto de Altos Estudios Nacionales:

http://repositorio.iaen.edu.ec/handle/24000/4470

En cuanto al analfabetismo digital se observa que es más relevante en las mujeres del área rural con un 43,2\%. Asimismo, los hombres del área rural presentan un mayor porcentaje de analfabetismo digital que los hombres de la zona urbana con un $34,4 \%$ frente a un $18,6 \%$ respectivamente. En tal virtud, se comprueba a nivel nacional que el analfabetismo digital es superior en las mujeres que en los hombres.

\section{Universidad Central del Ecuador}

Carreras de pregrado

La información que a continuación se analiza, muestra estadísticas de los estudiantes de pregrado de las facultades o sedes con carreras afines a las tecnologías de la información y la comunicación de la Universidad Central del Ecuador. Evidenciando que en las facultades que ofertan carreras duras como Electromecánica o Geología, las mujeres no representan la mayoría de su estudiantado a pesar de existir el acceso igualitario; en cambio, en aquellas facultades o sedes con carreras relacionadas al Comercio, Contabilidad, Comunicación Social, las mujeres superan a los hombres considerablemente, al igual que en las Ciencias Económicas y Ciencias Médicas. 


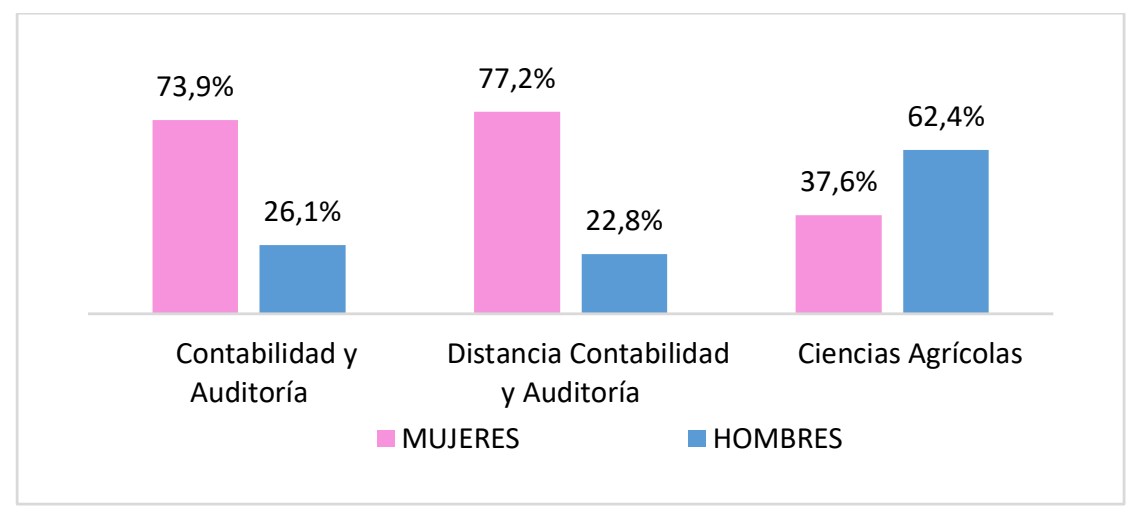

Figura 5. Porcentaje de estudiantes por carrera. Facultad de Ciencias Administrativas. Fuente: Dirección de Tecnologías de la Información y Telecomunicaciones - UCE (2013)

Se observa que en las carreras de la Facultad de Ciencias Administrativas la mayor demandada por mujeres es la de Contabilidad y Auditoría en sus dos modalidades, presencial y distancia, alcanzando porcentajes del $73,9 \%$ y $77,2 \%$ respectivamente; mientras que, la carrera de Ciencias Agrícolas, es predominantemente masculina con un $62,4 \%$ frente a un $37,6 \%$ femenino.

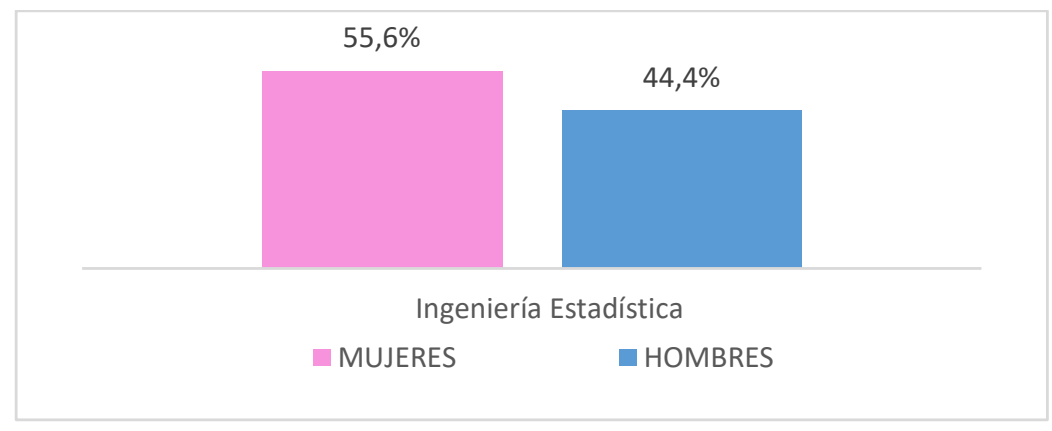

Figura 6. Porcentaje de estudiantes en la carrera de Ingeniería Estadística. Facultad de Ciencias Económicas.

Fuente: Dirección de Tecnologías de la Información y Telecomunicaciones - UCE (2013)

En la Carrera de Ingeniería Estadística las mujeres superan a los hombres con un 55,6\% frente a un $44,4 \%$ respectivamente. 


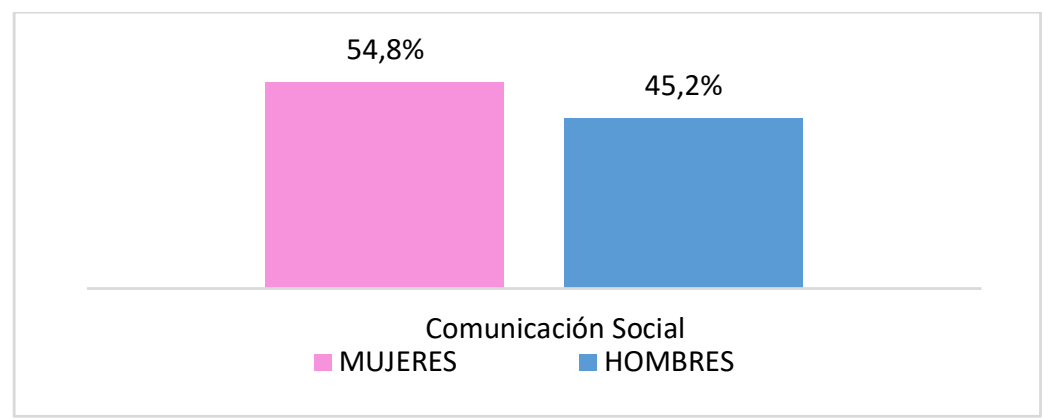

Figura 7. Porcentaje de estudiantes en la carrera de Comunicación Social.

Fuente: Dirección de Tecnologías de la Información y Telecomunicaciones - UCE (2013)

Las mujeres en el ámbito de la Comunicación Social son mayoría, lo cual se demuestra en el mayor acceso de estas a dicha carrera, un $54,8 \%$ frente a un $45,2 \%$ masculino.

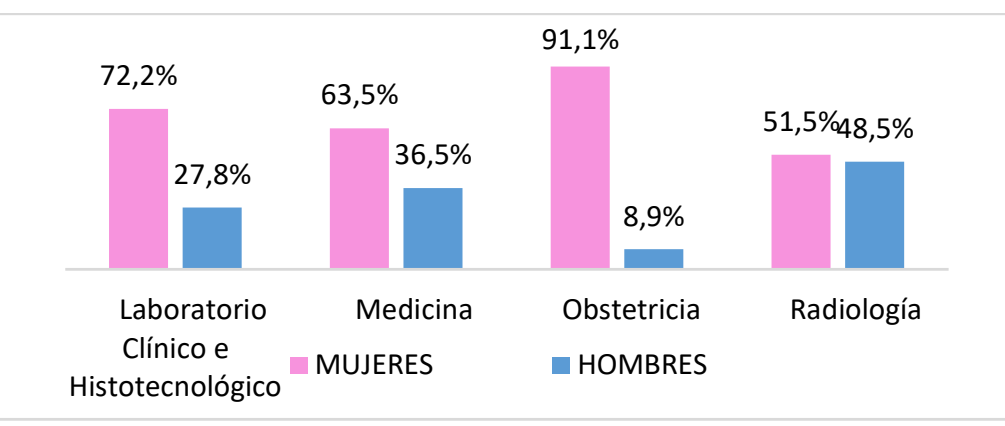

Figura 8. Porcentaje de estudiantes en las carreras de la Facultad de Ciencias Médicas. Fuente: Dirección de Tecnologías de la Información y Telecomunicaciones - UCE (2013)

Según los datos de la figura 8, en la Facultad de Ciencias Médicas las mujeres confirman su predominio frente a los hombres en todas sus carreras.

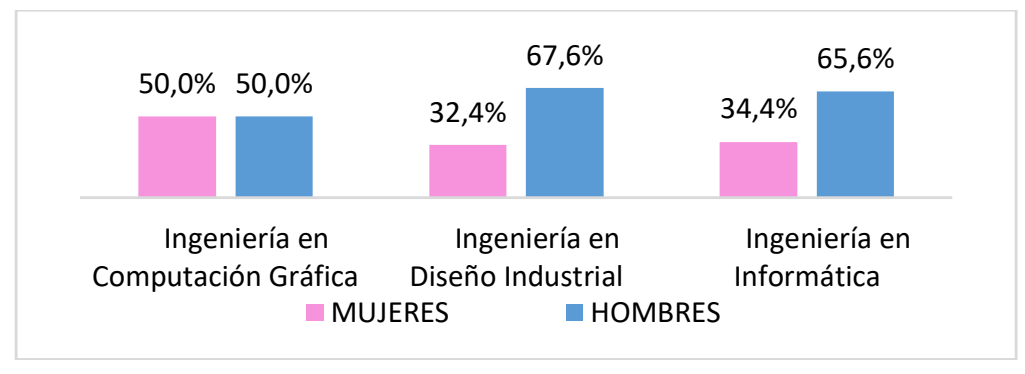

Figura 9. Porcentaje de estudiantes en las carreras de ingeniería. Facultad de Ciencias Físicas y Matemáticas.

Fuente: Dirección de Tecnologías de la Información y Telecomunicaciones - UCE (2013) 
La figura 9 muestra el predominio masculino en el caso de las Ingenierías, con porcentajes por encima de la media, comprobando la hipótesis de que las carreras consideradas duras no receptan mucho acceso femenino.

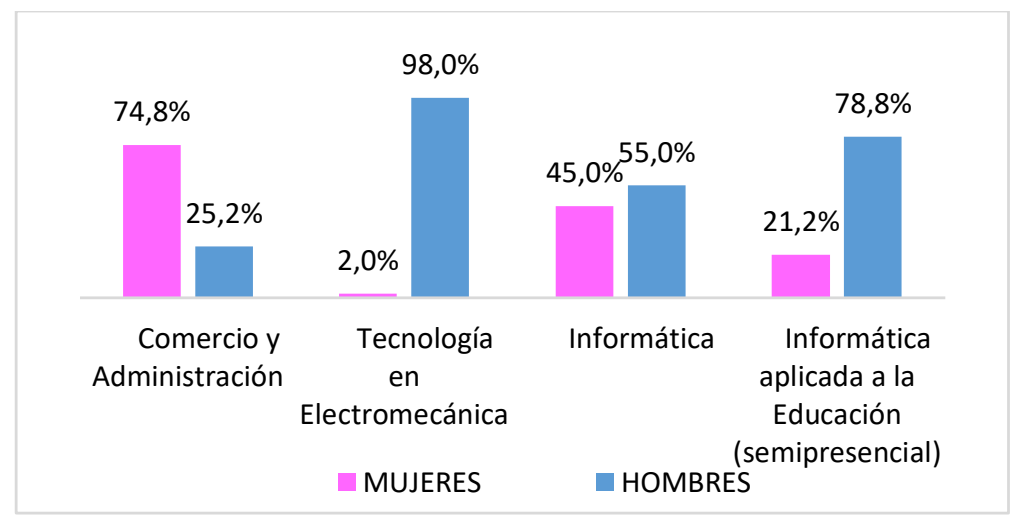

Figura 10. Porcentaje de estudiantes en las carreras de la Facultad de Filosofía, Letras y Ciencias de la Educación.

Fuente: Dirección de Tecnologías de la Información y Telecomunicaciones - UCE (2013)

En la Facultad de Filosofía, Letras y Ciencias de la Educación todas las carreras técnicas tienen un alto porcentaje de acceso masculino. Como se puede apreciar en la figura 10, el predominio femenino se sigue comprobando en carreras relacionadas al Comercio y Administración con un porcentaje del $74,8 \%$ frente a un $25,2 \%$ masculino.

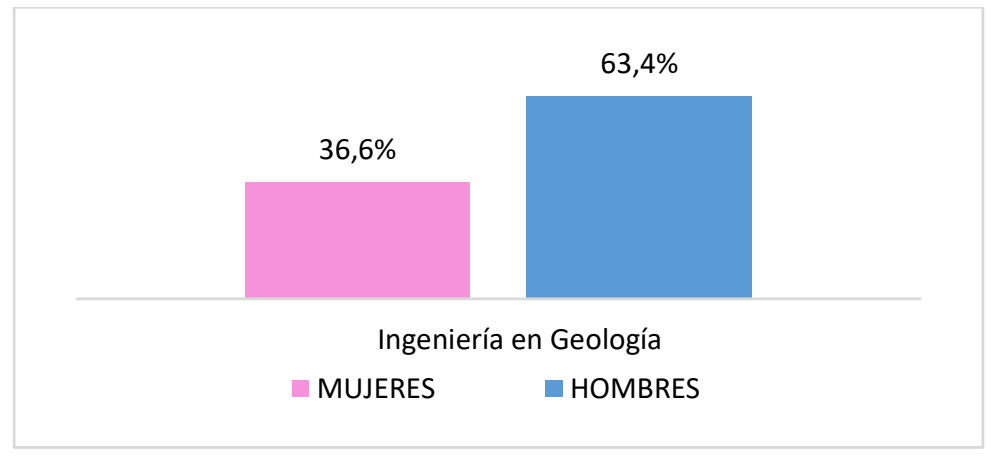

Figura 11. Porcentaje de estudiantes en la carrera de Ingeniería en Geología de la Facultad de Geología, Minas, Petróleo y Ambiental.

Fuente: Dirección de Tecnologías de la Información y Telecomunicaciones - UCE (2013) 
Haciendo un análisis con los datos de la figura 11, en la carrera de Geología, Minas, Petróleo y Ambiental se evidencia de forma muy marcada la preeminencia masculina con un $63,4 \%$ frente a un $36,6 \%$ femenino.

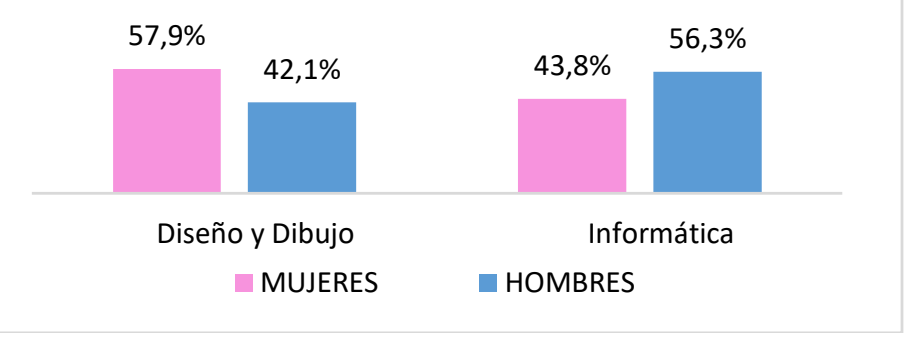

Figura 12. Porcentaje estudiantes en la carrera de Diseño y Dibujo e Informática. Sede Santo Domingo.

Fuente: Dirección de Tecnologías de la Información y Telecomunicaciones - UCE (2013)

En la Sede de Santo Domingo de la Universidad Central del Ecuador, la carrera de Diseño y Dibujo muestra la supremacía de las mujeres con un $57,9 \%$ frente a un $42,1 \%$ de los hombres. Mientras que en la carrera de Informática se evidencia la inversa, el predominio masculino es del $56,3 \%$ frente al femenino del $43,8 \%$, lo cual tiene relación con lo observado en la figura 10.

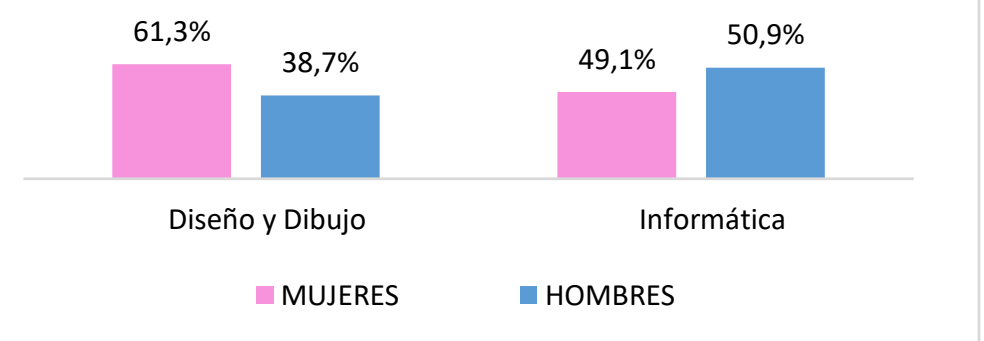

Figura 13. Porcentaje de estudiantes en las carreras de Diseño y Dibujo e Informática. Sede Galápagos.

Fuente: Dirección de Tecnologías de la Información y Telecomunicaciones - UCE (2013)

En la Sede de Galápagos de la Universidad Central del Ecuador, figura 13, se observa la misma tendencia de la figura 13 , que representa los datos de la Sede de Santo Domingo. 
En la carrera de Diseño y Dibujo el predominio de las mujeres con un $61,3 \%$ y en la carrera de Informática, el predominio masculino con un 50,9\%.

\section{Carreras de posgrado}

Las estadísticas siguientes corresponden a los estudiantes de la Universidad Central del Ecuador con postgrados afines a las tecnologías de la información y la comunicación, las cuales muestran coherencia con el pregrado. En Facultades como Arquitectura y Urbanismo, Ciencias Económicas, Ingeniería, Ciencias Físicas y Matemática y Ciencias Médicas - Especialidad en Radioterapia, el porcentaje de hombres es considerablemente superior al de las mujeres; mientras que en las de Filosofía, Letras y Ciencias de la Educación o Ciencias Médicas - Especialidad en Radiodiagnóstico e Imagen, sucede lo inverso, el porcentaje de mujeres que acceden a dichos posgrados es superior al de los hombres. Por lo que se comprueba que el rol de la mujer es preponderante en la elección de su formación profesional tanto de grado como de posgrado.

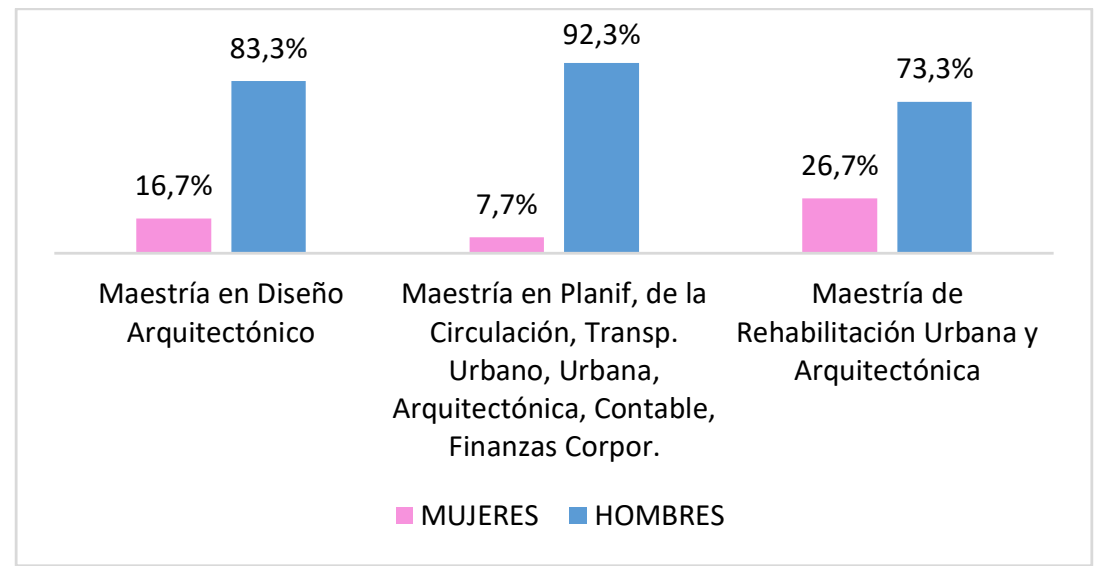

Figura 14. Porcentaje de estudiantes de la Maestrías de la Facultad de Arquitectura y Urbanismo.

Fuente: Dirección de Tecnologías de la Información y Telecomunicaciones - UCE (2013)

En las maestrías de la Facultad de Arquitectura y Urbanismo, figura 14, por ser considerada una carrera dura, la preeminencia masculina en las maestrías que oferta esta facultad es visiblemente marcada. Las diferencias porcentuales entre hombres y mujeres para cada maestría, de izquierda a derecha de la figura 15 , son de $66,6 \%, 84,6 \%$ y $46,6 \%$ respectivamente. 


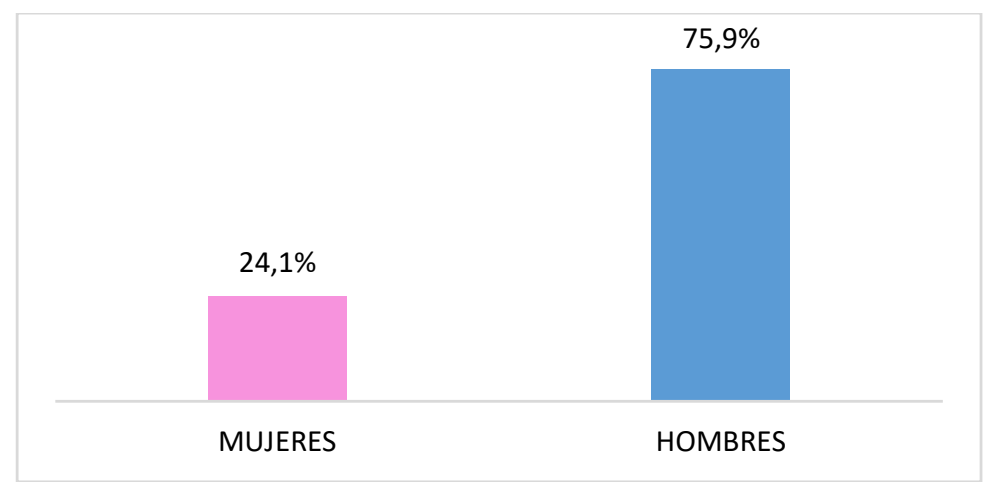

Figura 15. Porcentaje de estudiantes de la Maestría de Diseño y Evaluación de Proyectos. Facultad de Ciencias Económicas.

Fuente: Dirección de Tecnologías de la Información y Telecomunicaciones - UCE (2013)

En la maestría en Diseño y Evaluación de Proyectos, figura 15, ofertada por la Facultad de Ciencias Económicas, se observa la misma tendencia de la figura 14, el predominio en la participación de los hombres con un $75,9 \%$ frente a un $24,1 \%$ de las mujeres.

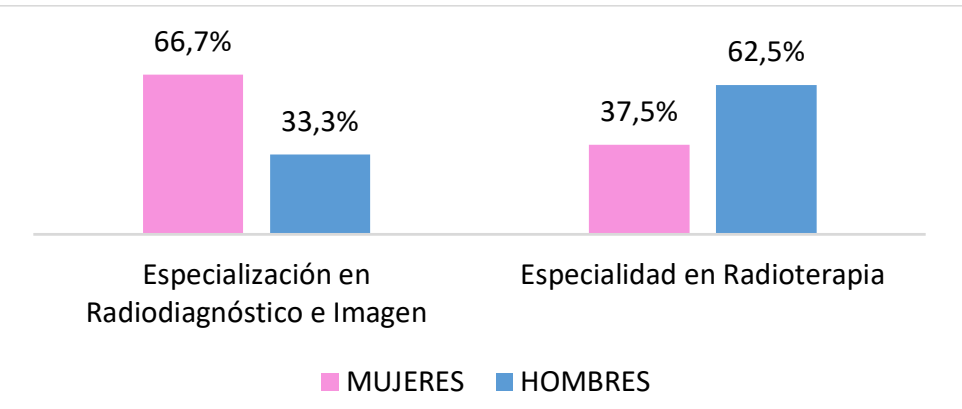

Figura 16. Porcentaje de estudiantes en los Programas de Posgrado. Facultad de Ciencias Médicas.

Fuente: Dirección de Tecnologías de la Información y Telecomunicaciones - UCE (2013)

En la figura 16, los posgrados de Ciencias Médicas se evidencia mayor participación femenil en la especialización en Radiodiagnóstico e Imagen con un 66,7\% mientras que en la especialidad en Radioterapia el dominio masculino representa un 62,5\%. 


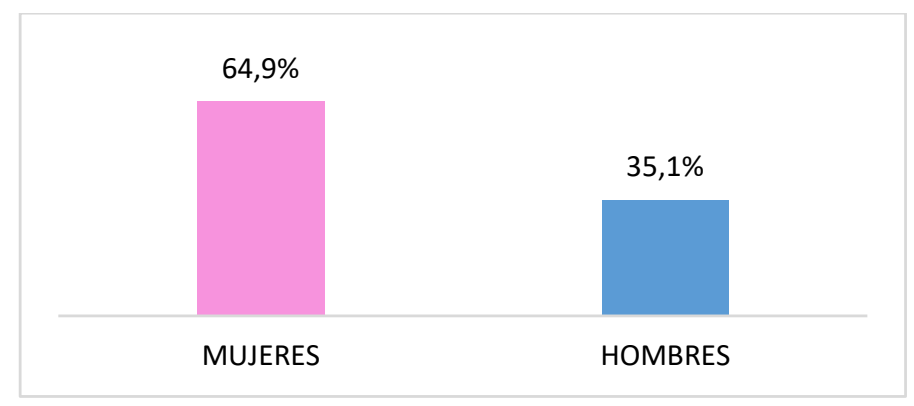

Figura 17. Porcentaje de estudiantes de la maestría de Diseño y Gestión de Proyectos Socioeducativos. Facultad de Filosofía, Letras y Ciencias de la Educación.

Fuente: Dirección de Tecnologías de la Información y Telecomunicaciones - UCE (2013)

En la figura 17, la maestría en Diseño y Gestión de Proyectos Socioeducativos campo profesional está ligado estrechamente a las mujeres, lo cual muestra una alta participación de éstas con un $64,9 \%$ frente a un $35,1 \%$ de los hombres.

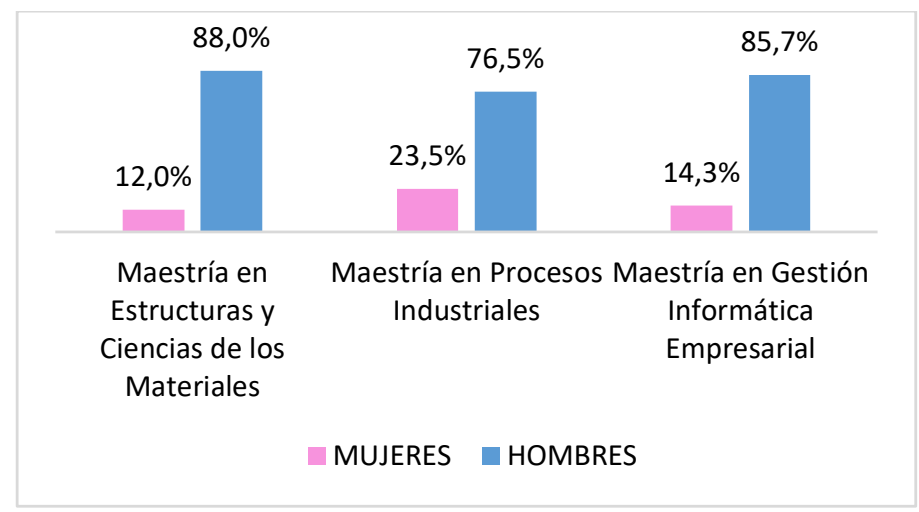

Figura 18. Porcentaje de estudiantes de las maestrías con base en Ingenierías. Facultad de Ingeniería, Ciencias Físicas y Matemáticas.

Fuente: Dirección de Tecnologías de la Información y Telecomunicaciones - UCE (2013)

En la figura 18, se evidencia en las maestrías con base en las Ingenierías se sigue la lógica del pregrado, la participación masculina supera a la femenina ampliamente. La Maestría en Estructuras y Ciencias de las Matemáticas y la Maestría en Gestión Informática Empresarial tienen una diferencia porcentual entre mujeres y hombres del $76 \%$ y $71,4 \%$ respectivamente, mientras que la Maestría en Procesos Industriales tiene una diferencia del $53 \%$. 


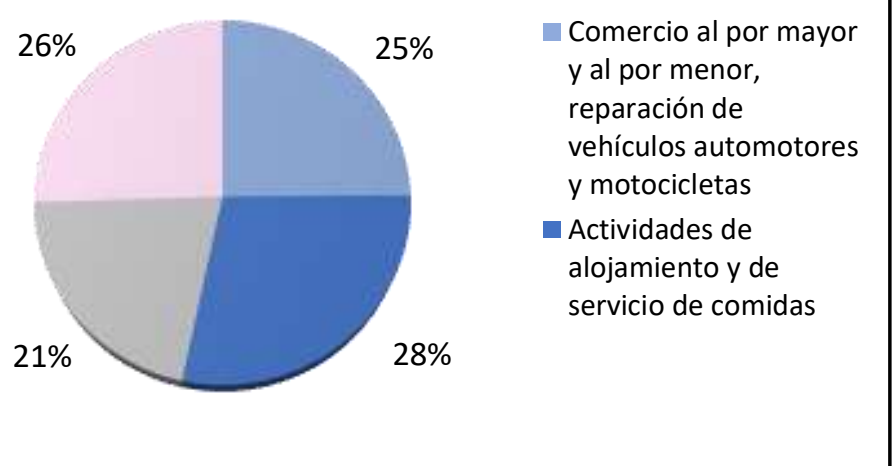

Figura 19. Porcentaje de mujeres por Propiedad del Establecimiento Económico según actividad principal.

Fuente: Censo Económico del Instituto Nacional de Estadísticas y Censos (2010)

En cuanto a la propiedad del negocio por actividad principal, las mujeres mayoritariamente lideran empresas relacionadas con los servicios de alojamiento, comidas, enseñanza, comercio e información y comunicación. Esta estadística de muestra la estrecha relación en la formación de las mujeres y su rol en la Economía del Cuidado, como se observa en la figura 19.

\section{Universidad de Guayaquil}

Carreras de pregrado

En las gráficas se muestra la situación de los estudiantes de Pregrado de la Universidad de Guayaquil con carreras afines a las Tecnologías de la Información y la Comunicación, evidenciando que en las ciencias duras como las Matemáticas o Ingenierías el porcentaje de hombres es considerablemente superior al de las mujeres. En cambio, en carreras tradicionales de Comercio y Administración, Economía, o Comunicación Social, las mujeres superan a los hombres. 


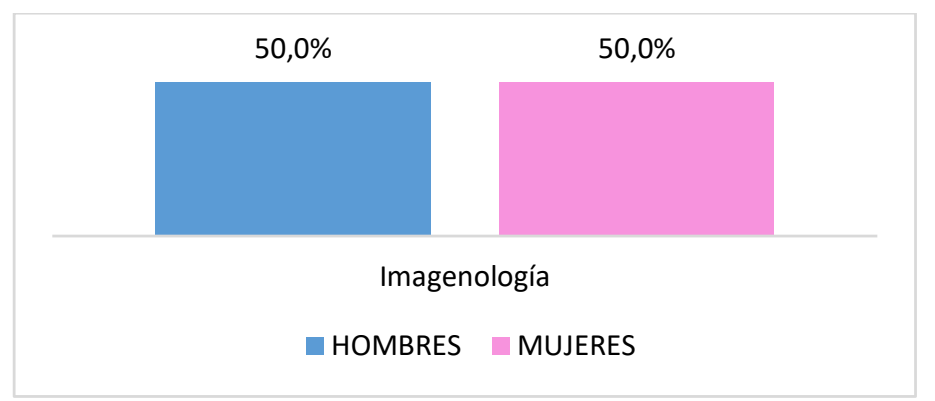

Figura 20. Porcentaje de Estudiantes en la carrera de Imagenología. Facultad de Ciencias Médicas.

Fuente: Centro de Cómputo de la Universidad de Guayaquil (2016)

En la figura 20 relacionada con la carrera de Imagenología se observa igualdad en el acceso entre hombres y mujeres.

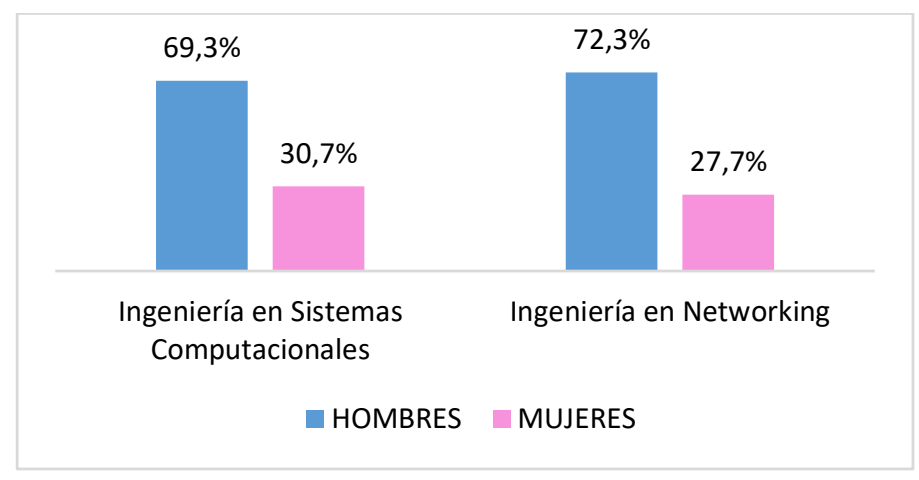

Figura 21. Porcentaje de estudiantes de las carreras de Ingeniería. Facultad de Ciencias Matemáticas y Físicas.

Fuente: Centro de Cómputo de la Universidad de Guayaquil (2016)

En la figura 21, las carreras de Ingeniería tienen un predominio de estudiantes varones. En la carrera de Ingeniería en Sistemas Computacionales el 69,3\% son hombres mientras que el $30,7 \%$ son mujeres, mientras en la carrera de Sistemas Computacionales el $72,3 \%$ son hombres frente al $27,7 \%$ que son mujeres. 


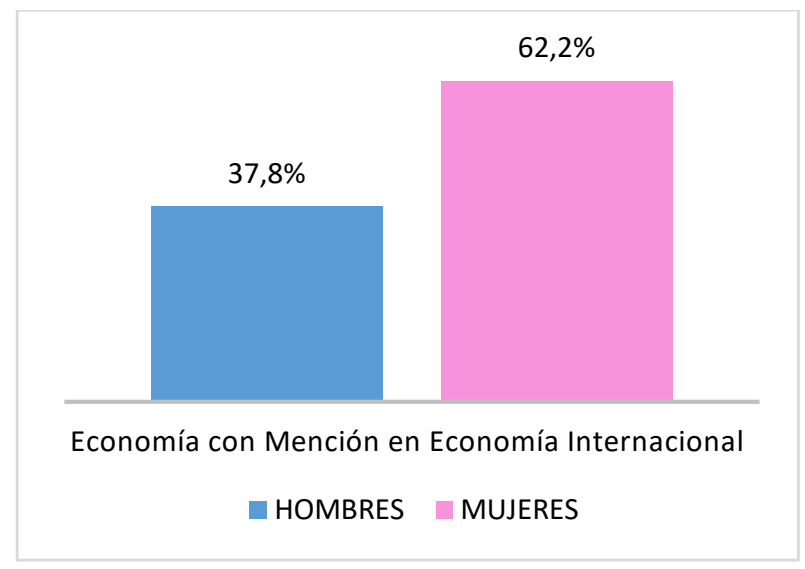

Figura 22. Porcentaje de Estudiantes de la carrera de Economía con Mención en Economía Internacional y Gestión del Comercio Exterior.

Fuente: Centro de Cómputo de la Universidad de Guayaquil (2016)

En la figura 22, se puede precisar que, en la Facultad de Ciencias Económicas con su carrera de Economía con Mención en Economía Internacional, la prevalencia es femenina con un $62,2 \%$ frente al $37,8 \%$ de los hombres, comprobándose una vez más que en las carreras relacionadas al comercio, el acceso de las mujeres es superior.

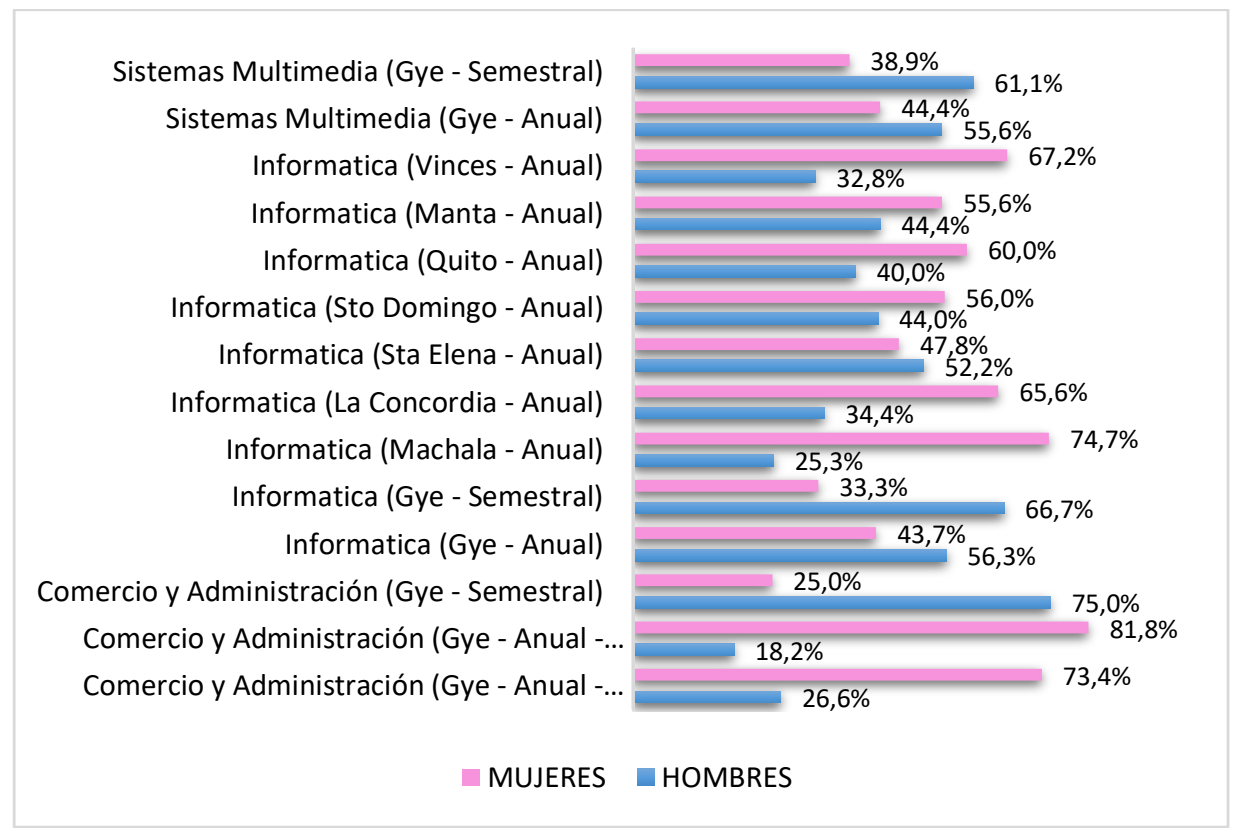

Figura 23. Comparación porcentual de la distribución de estudiantes en las carreras de la Facultad de Filosofía.

Fuente: Centro de Cómputo de la Universidad de Guayaquil (2016) 
Entre las carreras de la Facultad de Filosofía, las carreras de Informática y Comercio y Administración son preferentemente femeninas, como se observa en la figura 23 , mientras que, las de sistemas multimedia son más de tipo masculino, por ir más relacionadas a materias duras en su pensum académico.

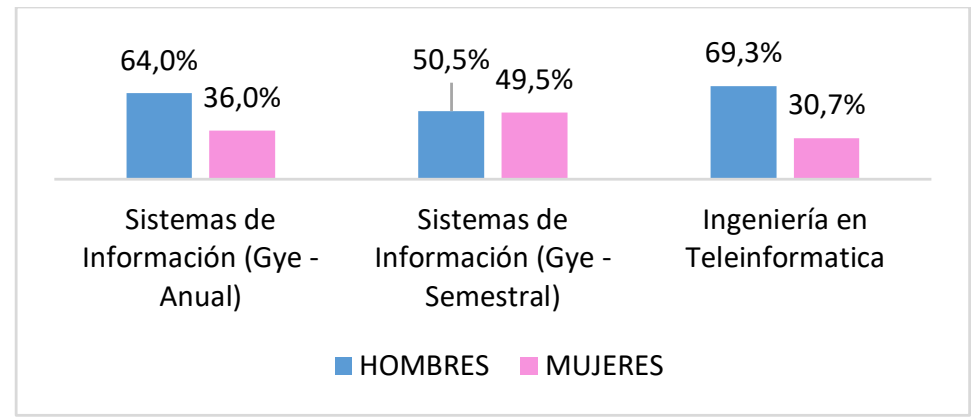

Figura 24. Porcentaje de estudiantes de las carreras de Sistemas de Información anual y semestral e Ingenia en Telecomunicaciones. Carrera de Ingeniería Industrial.

Fuente: Centro de Cómputo de la Universidad de Guayaquil (2016)

En la figura anterior se puede evidenciar que en esta facultad, las carreras son predominantemente masculinas por estar relacionadas a las Ingenierías, carreras duras con mayor acceso por parte de los hombres.

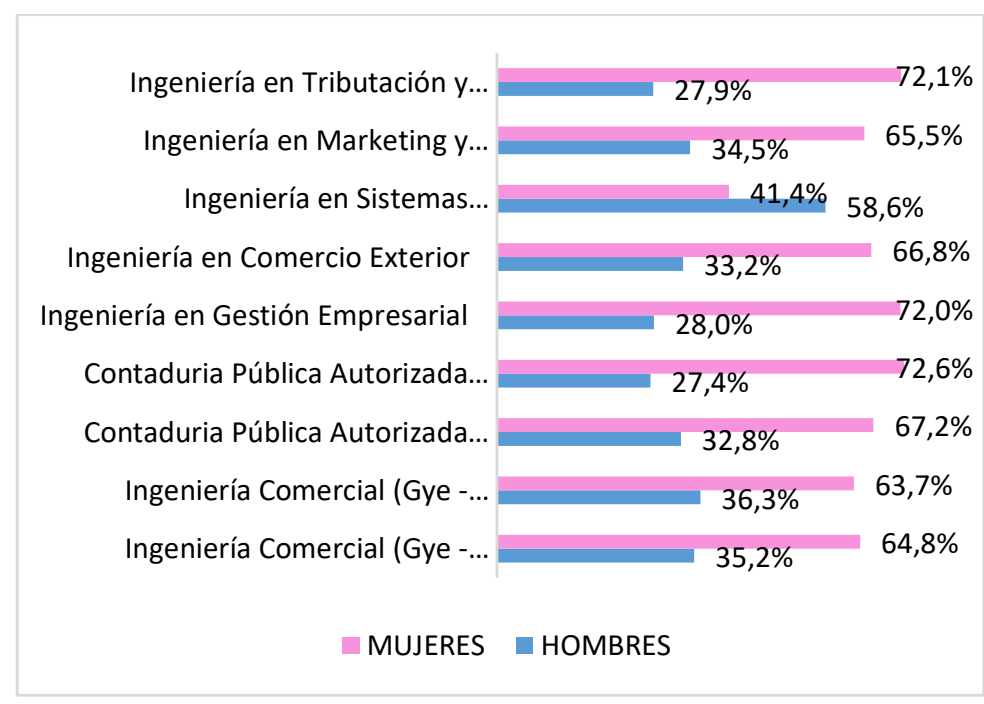

Figura 25. Porcentaje de estudiantes por carreras de la Facultad de Ciencias Administrativas.

Fuente: Centro de Cómputo de la Universidad de Guayaquil (2016). 
A diferencia de las Ingenierías no relacionadas a temas de Comercio y Administración, se puede comprobar en la figura 25 , que la preferencia femenina es marcada en el $90 \%$ de ellas.

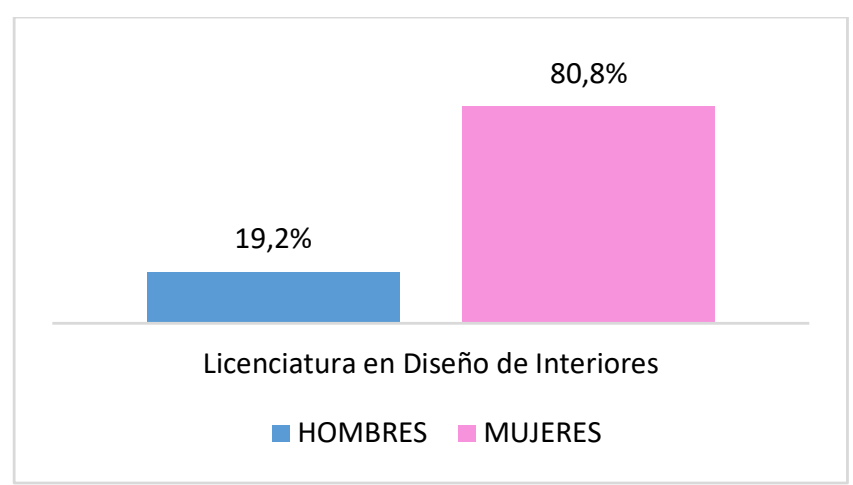

Figura 26. Porcentaje de estudiantes de la carrera de Licenciatura de Diseño de Interiores. Facultad de Arquitectura y Urbanismo.

Fuente: Centro de Cómputo de la Universidad de Guayaquil (2016)

En la figura 26, la Licenciatura en Diseño de Interiores también es un mercado cautivo del sexo femenino registrando un $80,8 \%$ de su acceso frente a un $19,2 \%$ masculino.

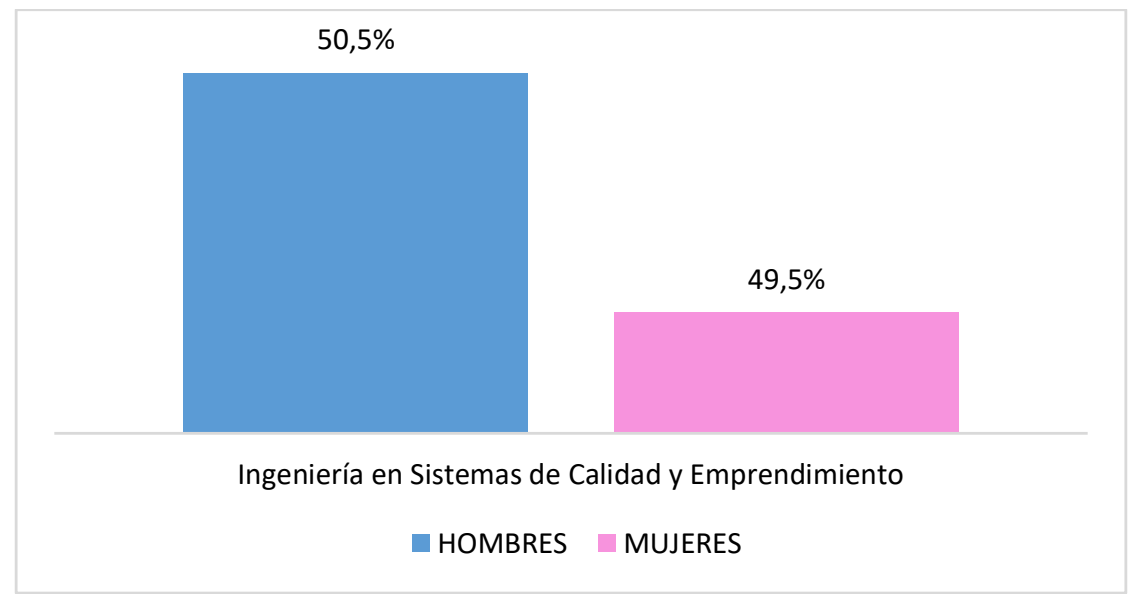

Figura 27. Porcentaje de estudiantes de la carrera Ingeniería en Sistemas de Calidad y Emprendimiento. Facultad de Ingeniería Química.

Fuente: Centro de Cómputo de la Universidad de Guayaquil (2016)

Al igual que el resto de las Ingenierías, en la figura anterior, se evidencia que la de Sistemas de Calidad y Emprendimiento tiene una ligera mayoría masculina del 50,5\% en relación a la femenina del $49,5 \%$. 


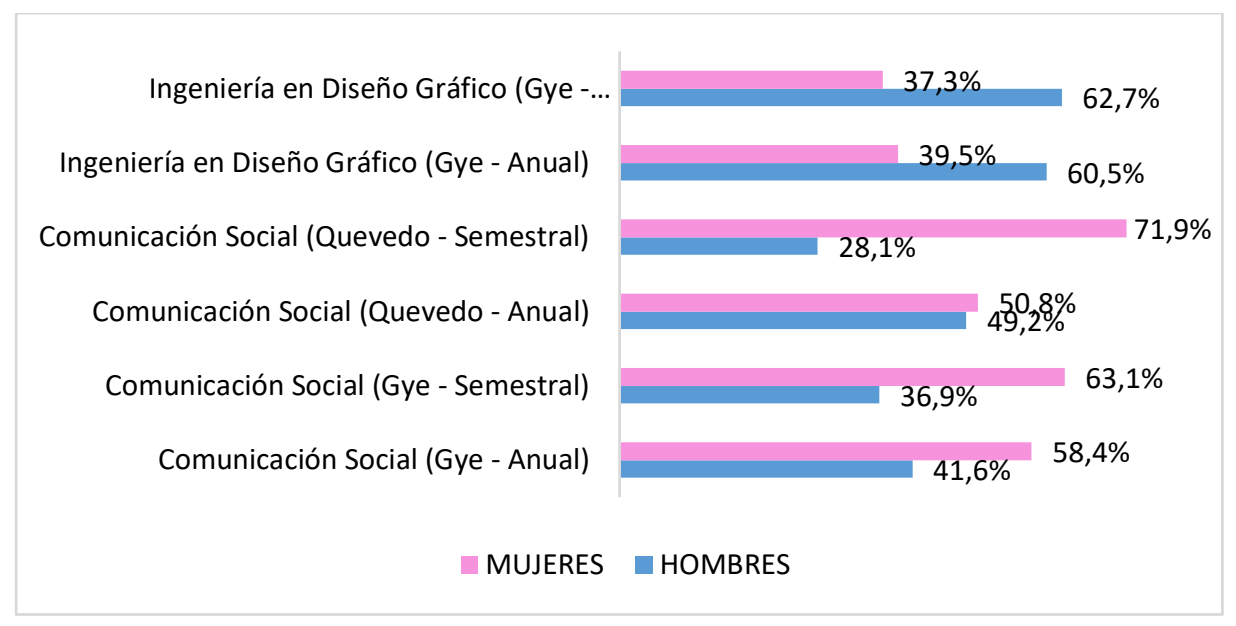

Figura 28. Porcentaje de estudiantes en las carreras de la Facultad de Comunicación Social.

Fuente: Centro de Cómputo de la Universidad de Guayaquil (2016)

Las carreras de la Facultad de Comunicación Social, en la figura 28, se puede constatar que son predominantemente ocupadas por las femeninas en un rango de 4 entre 6 , tanto que las Ingenierías en Diseño Gráfico comprueban su afinidad masculina con un 62,7\% y un $60,5 \%$ respectivamente.

A nivel nacional, el uso que se le da al internet tiene ligeras variaciones, entre el porcentaje de mujeres y hombres que utilizan la red. Para tal efecto, se analizan los datos que se han recopilado de la Encuesta Nacional de Empleo Desempleo y Subempleo - ENEMDU (2016), como se observa en la figura 29. 


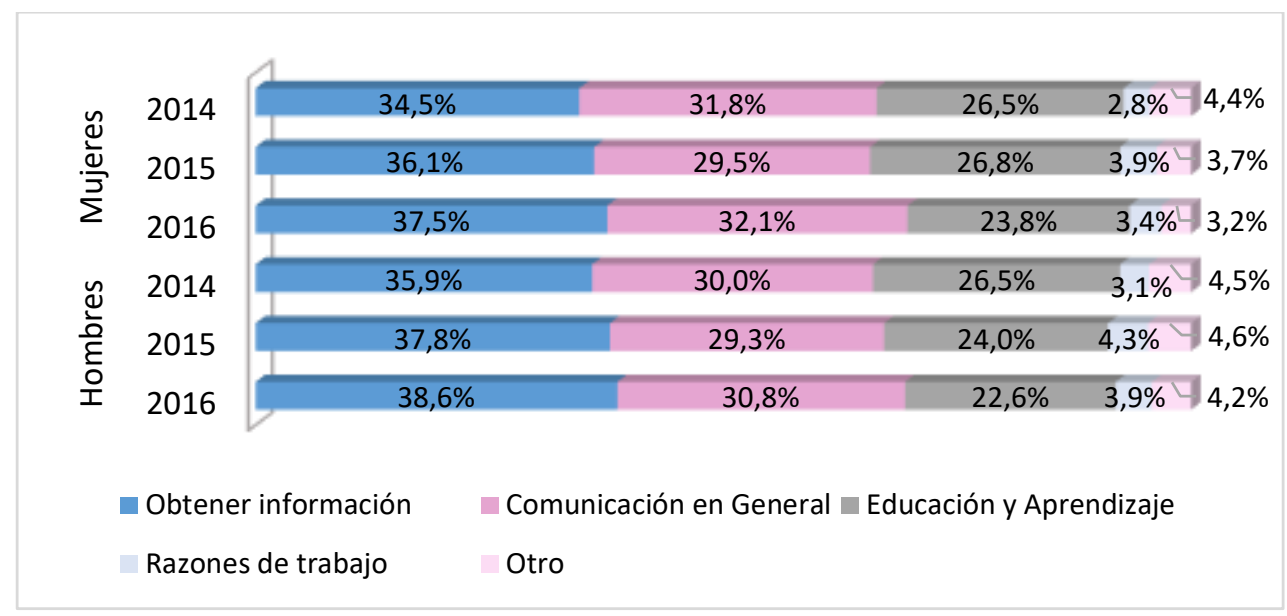

Figura 29. Razones de uso del internet por sexo.

Fuente: Encuesta Nacional de Empleo, Desempleo y Subempleo - ENEMDU (2012 2016)

Para el 2016 a nivel nacional (figura 29) el 38,6\% de los hombres usó Internet como fuente de información frente al 37,5\% de mujeres que lo utilizó por la misma razón, mientras el $30,8 \%$ de los hombres lo utilizó como medio de comunicación en general frente al 32,1\% de mujeres que lo utilizó por la misma razón. Las diferencias son muy pequeñas respecto a estos dos modos de uso para los dos tipos de género, 1,1\% y 1,3\% respectivamente. De la misma manera, si se observa el uso que se le da al internet por razones de educación y aprendizaje por un lado y las de trabajo por otro, se puede identificar significativamente una diferencia. En el período comprendido entre el 2014 y el 2016 se aprecia un promedio de uso por razones de educación y aprendizaje entre mujeres y hombres del $25,7 \%$ y $24,4 \%$ respectivamente. Por otro lado, si se observa el uso que se da al internet por razones de trabajo entre los dos géneros, se puede ver que existe en promedio 3,4\% de mujeres y $3,8 \%$ de hombres en esta variable.

Esta situación lleva a reflexionar el potencial de la generación que actualmente se encuentra en estudio, en cuanto a la aplicación de las TIC. 
Tabla 4. Encuestas sobre las tareas relacionadas con las info-habilidades por nivel de estudios.

\begin{tabular}{|c|c|c|c|c|c|c|c|}
\hline & $\begin{array}{l}\text { Tareas } \\
\text { relacionadas } \\
\text { con el PC }\end{array}$ & $\begin{array}{l}\text { 1era } \\
\text { etapa } \\
\text { secunda } \\
\text { ria }\end{array}$ & $\begin{array}{l}\text { 2da } \\
\text { etapa } \\
\text { secunda } \\
\text { ria }\end{array}$ & $\begin{array}{l}\text { Ed. } \\
\text { Universi } \\
\text { taria }\end{array}$ & $\begin{array}{l}\text { Tercer } \\
\text { Nivel }\end{array}$ & $\begin{array}{l}\text { Cuarto } \\
\text { Nivel } \\
\text { (Maestría) }\end{array}$ & $\begin{array}{l}\text { Cuarto } \\
\text { Nivel } \\
\text { (Doctorad } \\
\text { o) }\end{array}$ \\
\hline & $\begin{array}{l}\text { Copiar o mover } \\
\text { ficheros o carpetas }\end{array}$ & 100,0 & $65,4 \%$ & $82,1 \%$ & $82,5 \%$ & $100,0 \%$ & $100,0 \%$ \\
\hline & $\begin{array}{l}\text { Usar cortar y pegar en } \\
\text { un documento }\end{array}$ & $100,0 \%$ & $76,9 \%$ & $82,1 \%$ & $77,5 \%$ & $96,4 \%$ & $87,5 \%$ \\
\hline H & $\begin{array}{l}\text { Usar fórmulas } \\
\text { aritméticas simples en } \\
\text { una hoja de cálculo }\end{array}$ & $100,0 \%$ & $50,0 \%$ & $75,0 \%$ & $70,0 \%$ & $96,4 \%$ & $87,5 \%$ \\
\hline 0 & Comprimir ficheros & $100,0 \%$ & $34,6 \%$ & $55,4 \%$ & $60,0 \%$ & $85,7 \%$ & $75,0 \%$ \\
\hline $\begin{array}{l}\text { M } \\
\text { B }\end{array}$ & $\begin{array}{l}\text { Conectar o instalar } \\
\text { dispositivos como un } \\
\text { módem o una } \\
\text { impresora }\end{array}$ & $100,0 \%$ & $69,2 \%$ & $82,1 \%$ & $60,0 \%$ & $92,9 \%$ & $75,0 \%$ \\
\hline E & $\begin{array}{l}\text { Escribir un programa } \\
\text { usando un lenguaje de } \\
\text { programación }\end{array}$ & $50,0 \%$ & $15,4 \%$ & $32,1 \%$ & $22,5 \%$ & $46,4 \%$ & $37,5 \%$ \\
\hline & $\begin{array}{l}\text { Programar o utilizar } \\
\text { macros en office } \\
\text { (Word, Excel) } \\
\text { utilizando Visual Basic } \\
\text { for Aplication. }\end{array}$ & $0,0 \%$ & $38,5 \%$ & $42,9 \%$ & $55,0 \%$ & $60,7 \%$ & $50,0 \%$ \\
\hline & $\begin{array}{l}\text { Copiar o mover } \\
\text { ficheros o carpetas }\end{array}$ & $100,0 \%$ & $77,6 \%$ & $85,4 \%$ & $85,7 \%$ & $86,8 \%$ & $100,0 \%$ \\
\hline & $\begin{array}{l}\text { Usar cortar y pegar en } \\
\text { un documento }\end{array}$ & $100,0 \%$ & $75,5 \%$ & $79,8 \%$ & $82,1 \%$ & $86,8 \%$ & $100,0 \%$ \\
\hline
\end{tabular}




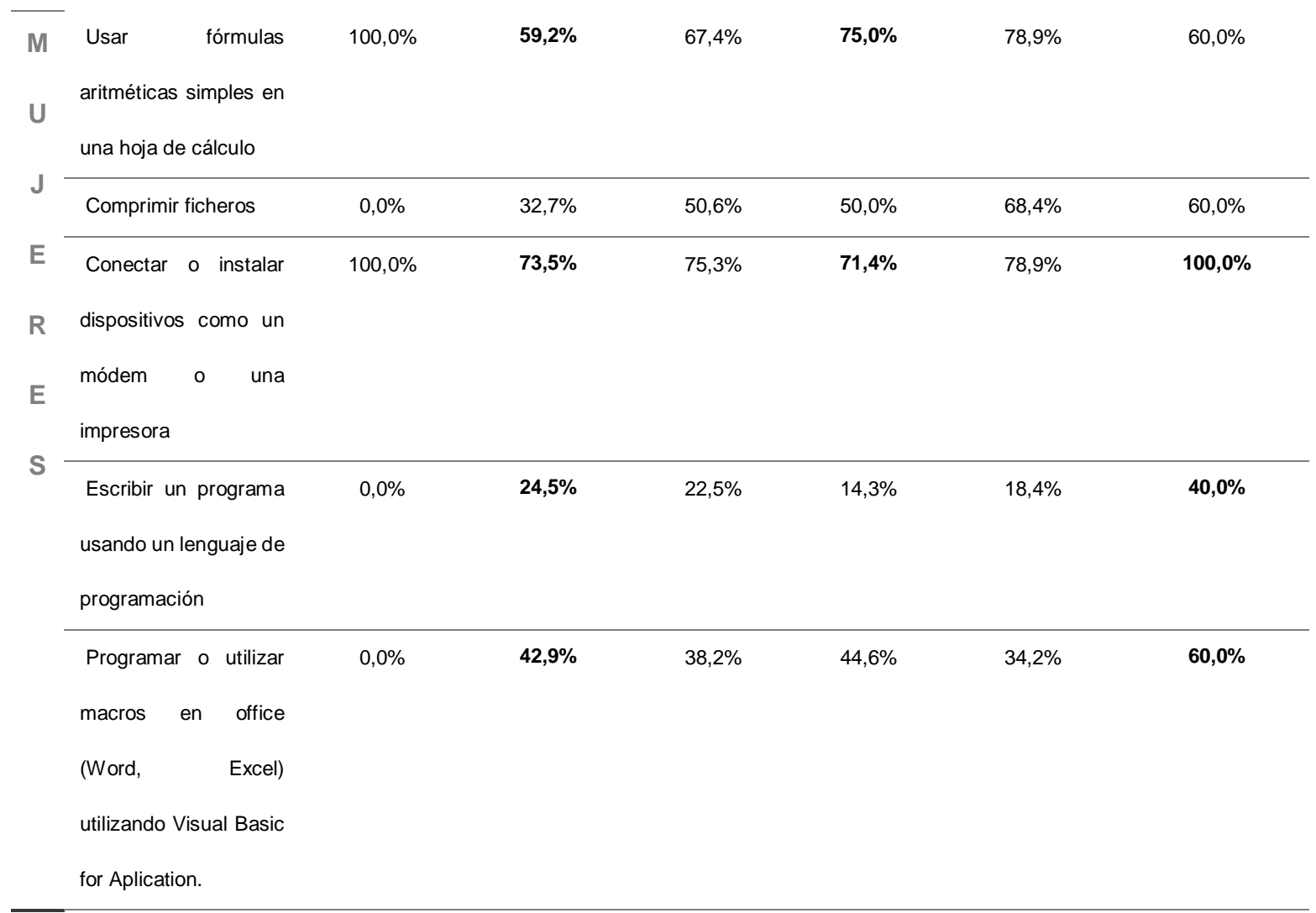

Fuente: Elaboración propia.

Los datos mostrados anteriormente ofrecen una perspectiva sobre las e-habilidades de las mujeres versus hombres por nivel de estudio, lo cual evidencia que las mujeres que se encuentran en segunda etapa secundaria o que tienen un título de tercer nivel, superan a los hombres en conocimientos y habilidades básicas y prácticas; asimismo se puede observar que las mujeres que tienen estudios de doctorado superan a los hombres en habilidades más avanzadas como la programación o utilización de macros en porcentajes entre el 2 y 10\%, no así en habilidades que conllevan más practicidad como la conexión o instalación de dispositivos cuya diferencia porcentual es del $25 \%$ superada por las mujeres. Sin embargo, es evidente que las habilidades de las mujeres de cuarto nivel (doctorado) decrecen en aspectos que requieren de habilidades matemáticas como el uso de fórmulas aritméticas simples, generalizándose este fenómeno para la mayoría de las mujeres según su etapa de estudio. 


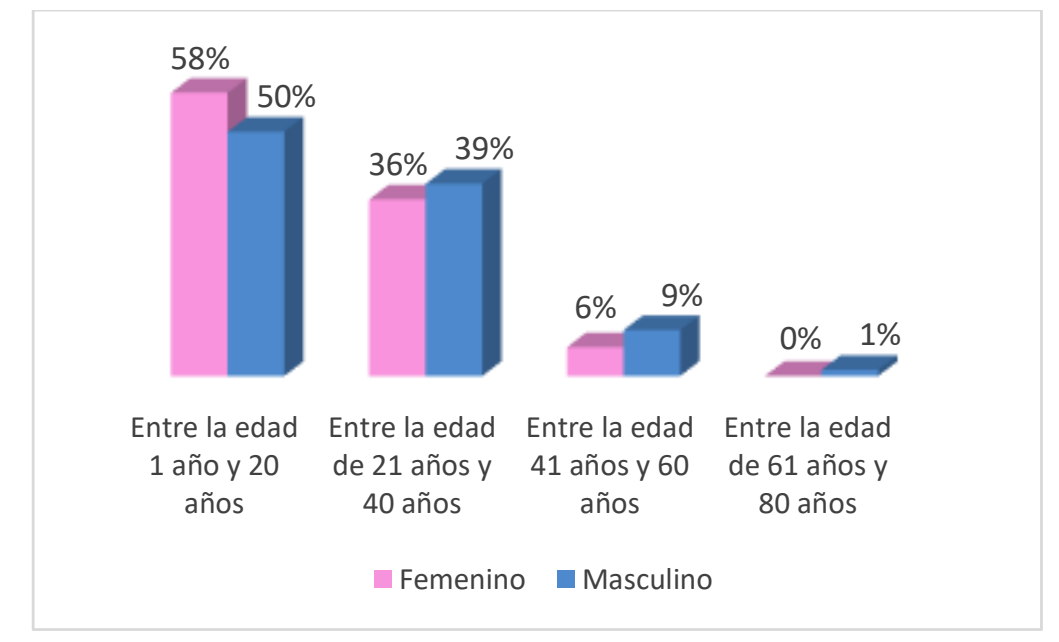

Figura 30. Encuesta sobre edad en la que se inició en el uso de internet. Fuente: Elaboración propia.

Se puede destacar el mayor impacto en la experiencia de uso del internet entre la población más joven, inclusive se denota que de este mismo grupo etario la mayor experiencia que se perfila es en las mujeres con un $58 \%$ de experiencia.

\section{DISCUSIÓN}

La importancia que se le está dando a la inclusión digital de las mujeres en América Latina, es tal, que en el año 2013 se realizó la XII Conferencia Regional sobre la Mujer para América Latina y el Caribe, del que nació el informe "Mujeres en la Economía Digital: Superar el Umbral de la desigualdad", lo cual indica que la frecuencia del uso la tecnología por las mujeres, en comparación con los hombres, va mucho más allá de un tema de infraestructura tecnológica o lenguaje, por lo que sus consecuencias son más graves de lo que se evidencia superficialmente (educación, formación profesional, empleo y acceso a ingresos).

Según el informe "How to connect the other half" (Cómo conectar a la otra mitad) encargado para la Global Commission on Internet Governance revela que 250 millones de latinoamericanas y latinoamericanos mayores de 15 años no accede ni es usuaria o usuario 
de internet regularmente, Galperín (2016). Otros estudios sobre "La inclusión financiera de las mujeres en América Latina" señala la necesidad de dinamizar un ecosistema institucional que contemple entre algunos aspectos el uso de tecnologías móviles o electrónicas como una herramienta de desarrollo de la mujer, su inclusión financiera y empoderamiento. CAF (2018).

En la Sexta Conferencia Ministerial sobre la Sociedad de la Información de América Latina y el Caribe, celebrada en Cartagena de Indias entre el 18 al 20 de abril de 2018, se aprobó la Agenda Digital para América Latina y el Caribe en su versión (eLAC2020), la misma que tiene como objetivo "ser un instrumento catalizador para la coordinación de los esfuerzos de cooperación regional en materia digital" (CEPAL, 2018). Este documento incluye 7 áreas de acción y 30 objetivos.

Dentro de las áreas de acción de la iniciativa regional, se destaca el número 7. Gobernanza para la sociedad de la información, en la que se encuentra el Objetivo 23:

Promover una perspectiva integral de igualdad de género en las políticas públicas de desarrollo digital, asegurando el pleno acceso y uso de las TIC a las mujeres y niñas, además del impulso de su participación y liderazgo en espacios públicos y privados de decisión. (CEPAL, 2018, p. 6)

Siguiendo el objetivo 7 de la eLAC2020 antes citado, los esfuerzos en el Ecuador se evidencian en las políticas públicas que se han diseñado para acortar la brecha digital y entre ellas las de género en cuanto a tecnología. Bajo la propuesta del gobierno ecuatoriano en delinear las Políticas Públicas del Sector de las Telecomunicaciones y de la Sociedad de la Información 2017 - 2021, las cuales fueron diseñadas considerando los avances de los dos sectores: problemáticas identificadas y nuevas oportunidades.

A continuación, se presenta en la figura 2, las políticas que se encuentran en el Registro Oficial (2017), donde se puede observar que el objetivo 3 que está dentro de las Políticas Públicas en el sector de las telecomunicaciones, orienta a que se universalice las TIC en la población y se propicie la inclusión con pertinencia de género, entre otros aspectos. De la 
misma manera el objetivo 2, que está inmerso en las Políticas Públicas para el desarrollo de la sociedad de la información propone construir el camino hacia la sociedad de la información y el conocimiento, centrada en la persona, inclusiva, integradora y orientada al desarrollo, tomando en cuenta sus capacidades y competencias.

En países como Colombia, la Encuesta de Monitoreo y Evaluación de Gobierno en Línea (2012) corroboró dos ideas fundamentales:

1. Que los hombres tienen mayor conocimiento que las mujeres a la hora de realizar trámites.

2. Que las mujeres utilizan en menor medida todos los dispositivos y la brecha más marcada se registra en los dispositivos móviles como teléfono celular, tabletas y otros.

Independiente de la brecha digital de género que también ha sido considerada en esta investigación, al análisis del uso que se le da al internet por parte de hombres y mujeres, el cual aporta un enfoque muy valioso donde la mujer utiliza el tiempo en el internet en actividades más funcionales, evidenciado en el grado de respuesta a la encuesta que tuvieron las mujeres versus hombres (por cada 10 personas encuestadas en línea 6 fueron mujeres y apenas 4 fueron hombres), considerar estos resultados y priorizar las ehabilidades de las mujeres, su practicidad en algunos aspectos y el uso que le da a las tecnologías, podría significar mayor bienestar socioeconómico. Asimismo, el estudio evidencia que el enfoque que actualmente tienen las mujeres para seleccionar sus actividades de educación, ocio, negocios o empleo, así como sus aptitudes para la eadministración, se relacionan con un mínimo grado de inclusión digital y falta de políticas diseñadas para la erradicación de los estereotipos sociales, heredados del capitalismo y la revolución industrial.

El análisis de las preferencias académicas en dos universidades del Ecuador, revela que la brecha de género se encuentra también relacionada con el bajo porcentaje de mujeres que estudian profesiones relativas a áreas del conocimiento como la Ingeniería de la Información y Tecnología de la Comunicación. Los resultados muestran diferencias significativas de género en estos aspectos; es decir, se evidencia la pervivencia de carreras masculinizadas y feminizadas, haciéndose imprescindible el diseño e implementación de 
políticas públicas de incentivo y promoción para la inserción de estudiantes mujeres, donde se visualiza menor participación.

Al analizar aspectos como las e-habilidades y la e-experiencia se puede observar que en muchos de los casos las mujeres superan a los hombres, el indicador de e-experiencia permite suponer que en el futuro las potencialidades del grupo etario más joven, en especial del género femenino podrían ser debidamente explotadas, siempre y cuando se diseñen las políticas adecuadas en cuanto al uso de software, hardware y otras herramientas para su desarrollo socioeconómico.

Frente a estos escenarios, se considera que la falta de educación tecnológica contribuye elocuentemente en el desempeño y empoderamiento de la mujer. En Ecuador, datos de la Comisión Económica para América Latina y el Caribe (CEPAL), evidencian que en el país el acceso a las nuevas tecnologías ha aumentado, y en muchos casos, la brecha de género se ha disminuido; sin embargo, consideramos la necesidad de que el país se plantee una agenda digital que incluya ejes de formación y orientación digital, que además, promueva la expansión de capacidades en las mujeres; ya que aunque se evidencia disminución de la brecha digital, en el sentido de que las diferencias de acceso al internet entre hombres y mujeres son mínimas, no pasa igual con el uso de las herramientas tecnológicas.

\section{CONCLUSIONES}

En el marco de esta investigación, es necesario acotar que las políticas deben apuntar a la consideración de la mujer como un sujeto no meramente reproductivo sino como un sujeto que a pesar de ser catalogado como no disponible, puede aportar al desarrollo socioeconómico del país desde sus ideas y capacidad de trabajo, es decir, un sujeto productivo.

Se estima que las oportunidades deben ser alcanzadas por todos sin distinción de género, más aún cuando se promueven relaciones de equidad en términos del desarrollo para ambos sexos. 
En cuanto a la inclusión de las TIC para el desarrollo, se debe aún insistir en el fortalecimiento del conocimiento y aplicación de herramientas tecnológicas para dar crecimiento al uso de tecnologías por parte del género femenino. Si bien es cierto que entre hombres y mujeres no existe mayor diferencia en el acceso, el problema se centra en el dominio de herramientas informáticas especializadas.

En el proceso de construcción de la agenda para la e-inclusión de la mujer, se puede sugerir que se incorporen políticas públicas orientadas a fomentar el interés y desarrollo de destrezas en el uso de tecnologías de la información y comunicación, así como también políticas enfocadas hacia las habilidades y conocimientos, que son propias de su cultura por herencia o educación, para ser explotados con el apoyo de las TIC. Esta situación supone que la mujer pueda explotar creativamente habilidades y conocimientos como base para su desarrollo profesional y familiar y en la cual el gobierno ecuatoriano debe enfocarse en señalar la ruta conforme a la estrategia regional de la eLAC 2020.

\section{REFERENCIAS BIBLIOGRÁFICAS}

Aguinaga, M., Lang, M., Mokrani D. \& Santillana, A. (2012). Pensar desde el feminismo: Críticas y alternativas al desarrollo. En Lang, M. \& Mokrani, D. (Ed.), Más allá del desarrollo (pp. 55-82). Quito, Ecuador: Abya-Yala. Recuperado de https://ebookcentral.proquest.com

Aponte, E. (2008). Desigualdad, Inclusión y equidad en educación superior en América Latina y el Caribe: Tendencias y escenario alternativo en el horizonte 2021. Gazzola, A. y Didriksson, A. (Eds.). Tendencias de la educación Superior en América Latina y el Caribe. (pp. 113 - 154) UNESCO-IESALC.

Arredondo, F., Maldonado, V. \& Velázquez, L. M. (2013). Liderazgo femenino e Innovación social. Monográfico Perspectiva de Género en Latinoamérica: Políticas Públicas, Educación, Salud, Familia, Participación Política. Economía y Trabajo. Revista Nuevo Mundo de Estudios Latinoamericanos, 8(13), 207-226.

Aznar, J. \& Navarro, J. (2012). Desafíos para el desarrollo personal, laboral y familiar de las mujeres rurales del arco mediterráneo. En Escrig, G. G., Ortí, P. M. J., \& 
Beltran, B. R. (Eds.). (2012). VIII Actas Congreso. El género de la economía o la economía de género. Recuperado de https://ebookcentral.proquest.com

Berrío, C.; Marín, P.; Ferreira da Silva, E.; das Chagas, E. (2017). Desafios da Inclusão Digital: antecedentes, problemáticas e medição da Exclusão Digital de Gênero. Psicología, Conocimiento y Sociedad, 7(2), 162-198. https://dx.doi.org/10.26864/pcs.v7.n2.8

Bethencourt, L. (2012). La Feminización de la fuerza de trabajo no garantiza la reducción de la brecha de género: el caso venezolano. Revista Venezolana de Estudios de la Mujer. 2012. VOL. 17/N³8. Pp.17-38. ISSN: 2244-7660. Recuperado de http://saber.ucv.ve/ojs/index.php/rev_vem/article/view/1991/1894

BID. (2017). Informe anual del Índice de Desarrollo de la Banda Ancha en América Latina y el Caribe IDBA 2016. Obtenido de Banco Interamericano de Desarrollo:

\section{http://dx.doi.org/10.18235/0000642}

BID. (2018). Conectividad. Recuperado el 7 de mayo de 2018, del Sitio Web del Banco Interamericano de Desarrollo: https://www.iadb.org/es/mercadosfinancieros/conectividad

Castaño, C., \& Webster, J. (2014). Entender el género en la ciencia y la tecnología. En Castaño, C., \& Webster, J. (Ed.), Género, ciencia y tecnologías de la información. (pp. 23-60). Barcelona, España: Editorial UOC. Recuperado de https://ebookcentral.proquest.com

CEPAL. (20 de abril de 2018). eLAC 2020 Sexta Conferencia Ministerial sobre la Sociedad de la Información de América Latina y el Caribe. Obtenido de eLAC2020: https://conferenciaelac.cepal.org/6/sites/elac2020/files/cmsi.6_agenda_digital.pdf

CEPAL. (2018). eLAC 2020 Sexta Conferencia Ministerial sobre la Sociedad de la Información de América Latina y el Caribe. Recuperado de e-LAC2020: https://conferenciaelac.cepal.org/6/es/documentos/agenda-digital-america-latinacaribe-elac2020

CEPAL. (2018). Estado de la banda ancha en América Latina y el Caribe. Santiago: Naciones Unidas.

CEPAL. (26 de mayo de 2015). Agenda digital de América Latina y el Caribe (eLAC) fue premiada en Cumbre Mundial sobre la Sociedad de la Información. Recuperado el 5 de agosto de 2018, de Sección Noticias: 
https://www.cepal.org/es/noticias/agenda-digital-de-america-latina-elac-fuepremiada-en-cumbre-mundial-sobre-la-sociedad-de

Ferreira, C., García, K., Macías, L., Pérez, A. \& Tomsich C. (2013). Mujeres y hombres del Ecuador en Cifras III. Serie información estratégica. Comisión de Transición hacia la Definición de la Institucionalidad Pública que garantice la Igualdad entre Hombres y Mujeres. Instituto Nacional de Estadística y Censos. ONU Mujeres. 2013. Recuperado de http://www.ecuadorencifras.gob.ec/wpcontent/descargas/Libros/Socioeconomico/Mujeres_y_Hombres_del_Ecuador_en_ Cifras_III.pdf.

García, M (2015). Discriminación femenina en el ámbito laboral: aspectos descriptivos, prescriptivos y contextuales de los estereotipos de género. Tesis doctoral, Universidad Nacional de Educación a distancia, España.

INEC. (2016). Tecnologías de la Información y Comunicaciones (TIC'S) 2016.

Mámedio da Costa, C., De Mattos, C. A., \& Cuce, M. R. (2007). Estrategia PICO para la construcción de la pregunta de investigación y la búsqueda de evidencias. Rev Latinoam Enfermagem [Internet], 15(3).

Ministerio de Telecomunicaciones y Sociedad de la Información. (2016). Plan Nacional de Telecomunicaciones y Tecnologías de Información del Ecuador 2016-2021. Obtenido de Sitio Web del Ministerio de Telecomiunicaciones y Sociedad de la Información del Ecuador: https://www.telecomunicaciones.gob.ec/wp-content/uploads/2016/08/Plande-Telecomunicaciones-y-TI..pdf.

Registro Oficial. (2017). Políticas Públicas del Sector de las Telecomunicaciones y de la Sociedad de la Información 2017 - 2021. Registro Oficial No. 15, pp. 14-19. Quito, Ecuador.

Restrepo, F. B., \& Márquez, I. D. (octubre de 2013). La Economía Naranja: Una oportunidad infinita. Recuperado de Banco Interamericano de Desarrollo, BID: https://publications.iadb.org/handle/11319/3659?locale-attribute=es

SENPLADES. (2014). Estrategia Nacional para la lgualdad y erradicación de la pobreza. Quito, Ecuador. 
Sharma y Arese (2016). Connected Society: Inclusión digital en América Latina y el Caribe. En Asociación Global de todo el Ecosistema Móvil (GSMA). Recuperado de https://www.gsmaintelligence.com

Torres, M. (2012). Estado social y sujeto productivo. un apunte constitucional desde la perspectiva de género. En Escrig, G. G., Ortí, P. M. J., \& Beltran, B. R. (Eds.). (2012). VIII Actas Congreso. El género de la economía o la economía de género. Recuperado de https://ebookcentral.proquest.com

Versozi, C., Muñoz, A. \& Versozi, P. (2017). Economía del conocimiento e Innovación. En Instituto de Investigaciones Económicas y Políticas (IIEPS) UG (Comp.) Retos y Perspectivas del Desarrollo Económico en el Ecuador y América Latina TOMO II. (pp. 474 - 483) CIIDE - ECUADOR. 